\title{
Exploring Interacting Topological Insulators with Ultracold Atoms: The Synthetic Creutz-Hubbard Model
}

\author{
J. Jünemann, ${ }^{1,2}$ A. Piga, ${ }^{3}$ S.-J. Ran, ${ }^{3}$ M. Lewenstein,,${ }^{3,4}$ M. Rizzi, ${ }^{1}$ and A. Bermudez ${ }^{5}$ \\ ${ }^{1}$ Johannes Gutenberg-Universität, Institut für Physik, Staudingerweg 7, 55099 Mainz, Germany \\ ${ }^{2}$ MAINZ-Graduate School Materials Science in Mainz, Staudingerweg 9, 55099 Mainz, Germany \\ ${ }^{3}$ ICFO-Institut de Ciencies Fotoniques, The Barcelona Institute of Science and Technology, \\ 08860 Castelldefels (Barcelona), Spain \\ ${ }^{4}$ ICREA, Lluis Companys 23, 08010 Barcelona, Spain \\ ${ }^{5}$ Department of Physics, Swansea University, Singleton Park, Swansea SA2 8PP, United Kingdom \\ and Instituto de Física Fundamental, IFF-CSIC, Madrid E-28006, Spain \\ (Received 21 December 2016; revised manuscript received 10 August 2017; published 27 September 2017)

\begin{abstract}
Understanding the robustness of topological phases of matter in the presence of strong interactions and synthesizing novel strongly correlated topological materials lie among the most important and difficult challenges of modern theoretical and experimental physics. In this work, we present a complete theoretical analysis of the synthetic Creutz-Hubbard ladder, which is a paradigmatic model that provides a neat playground to address these challenges. We give special attention to the competition of correlated topological phases and orbital quantum magnetism in the regime of strong interactions. These results are, furthermore, confirmed and extended by extensive numerical simulations. Moreover, we propose how to experimentally realize this model in a synthetic ladder made of two internal states of ultracold fermionic
\end{abstract} \\ atoms in a one-dimensional optical lattice. Our work paves the way towards quantum simulators of \\ interacting topological insulators with cold atoms.
}

DOI: 10.1103/PhysRevX.7.031057

Subject Areas: Atomic and Molecular Physics, Quantum Information

\section{INTRODUCTION}

Topological features of quantum many-body systems provide a new paradigm in our understanding of the phases of matter [1], and give rise to a promising avenue towards fault-tolerant quantum computation [2]. From a condensedmatter perspective, such features lead to exotic ground states beyond the conventional phases of matter, which are typically understood by the principle of symmetry breaking and the notion of a local order parameter. On the contrary, these exotic states can be characterized by only certain topological properties.

The integer quantum Hall effect, which is a paradigmatic example of such peculiar phases [3], requires the introduction of a topological invariant to describe the different plateaus and their associated transverse conductivities [4]. Another interesting property of this state of matter is the bulk-boundary correspondence, which relates such a topological conductivity, a bulk property, to the existence of current-carrying edge states localized within the boundaries of the system [5]. Although the bulk of an integer quantum Hall sample appears as a trivial band insulator, its

Published by the American Physical Society under the terms of the Creative Commons Attribution 4.0 International license. Further distribution of this work must maintain attribution to the author(s) and the published article's title, journal citation, and DOI. boundary corresponds to a holographic chiral liquid where interactions merely renormalize the edge-state Fermi velocity [6].

As realized in a series of seminal works [7-9], these remarkable properties are not unique to quantum Hall samples subjected to strong magnetic fields. Instead, they arise in various models with different symmetries and in different dimensions [10], the so-called topological insulators and superconductors [11], which also lead to the notion of symmetry-protected topological phases in the context of topological order [1]. Remarkably enough, some of these models have turned out to be accurate descriptions of real insulating materials [11-13] and promising candidates to account for observations in proximitized superconducting materials [14]. This has positioned the subject of topological insulators and superconductors not only at the forefront of academic research, but also at the focus of technological applications, such as topological quantum computation with Majorana fermions [2].

Despite this success, (i) there are still several paradigmatic models of topological models whose connection to real materials remains unknown, or even seems quite unlikely, as it occurs for the Hofstadter model with magnetic fluxes on the order of the flux quantum [15], or the Haldane model [7]. Moreover, (ii) most of the topological materials explored in the laboratory so far do not display important electronic correlation effects [16]. This is rather unfortunate in view of the richness of the 
fractional quantum Hall effect [17], where such correlations are responsible for a plethora of exotic topological phases of matter.

In the present work, rather than considering real materials, we are concerned with the so-called synthetic quantum matter in atomic, molecular, and optical (AMO) platforms, more particularly, with ultracold gases of neutral atoms trapped in periodic potentials made of light, i.e., optical lattices [18]. The ever-improving experimental control over these quantum many-body systems has already allowed to design their microscopic Hamiltonian to a great extent. In this way, it is possible to target interesting condensed-matter models, some of which are still lacking unambiguous experimental feedback from experiments with real materials $[19,20]$, as occurs for the bosonic [21-23] and fermionic [24-26] Hubbard models. The initial interest in opticallattice implementations of integer quantum Hall phases [27], and other time-reversal invariant topological insulators $[28,29]$, has risen considerably in recent years due to the experimental progress [30,31]. In particular, elusive topological systems, such as the Hofstadter [15] or the Haldane [7] models, have been realized in experiments with bosonic [32] and fermionic [33] ultracold atoms, respectively. The possibility of including two or more different atomic states or species, controlling their interactions via Feshbach resonances [34], may eventually lead to the experimental test of correlation effects in these topological states.

Here, we introduce a variant of the quasi-onedimensional Creutz topological insulator [35] and study the effect of repulsive Hubbard-type interactions on the topological phase. This model, which we refer to as the imbalanced Creutz-Hubbard ladder, is readily implementable with ultracold fermions in intensity-modulated optical lattices. We argue that such a model has all the required ingredients to become a workhorse in the study of strongly correlated topological phases in AMO setups. Indeed, two accessible AMO ingredients such as (i) a simple Zeeman shift between the atomic internal states, which yields a leg imbalance in the ladder, and (ii) on-site repulsive contact interactions tuned by some Feshbach resonances, which lead to Hubbard-type rung interactions in the ladder, can be employed to access the rich phase diagram of this model (see Fig. 1), which was widely uncharted prior to our study. Starting from a flat-band regime, we show that the imbalance and the interactions lead to a competition between a topological phase and two different phases of orbital quantum magnetism. At large interaction strength, a long-range in-plane ferromagnetic order arises, related to the symmetry-broken phase of an orbital quantum Ising model (QIM), while the Zeeman imbalance then drives a standard quantum phase transition in the Ising universality class towards an orbital paramagnetic phase. In order to understand the model away from this limit, we introduce two new methods based on mappings onto models of quantum magnetism and quantum impurity physics. These

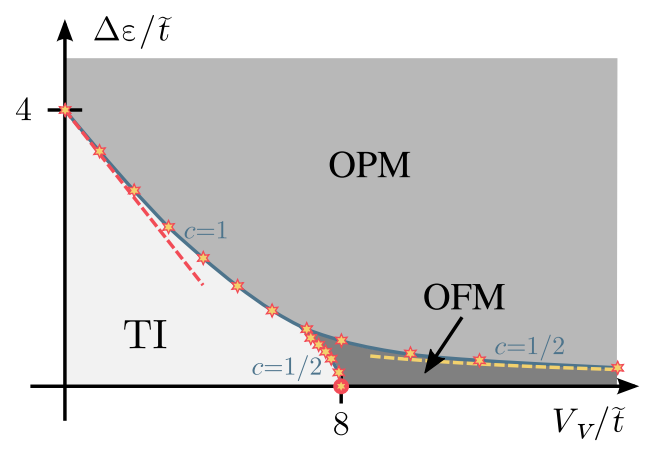

FIG. 1. Phase diagram of the imbalanced Creutz-Hubbard ladder. Phase diagram displaying a topological insulator (TI) phase and a pair of nontopological phases: an orbital phase with long-range ferromagnetic Ising order (OFM) and an orbital paramagnetic phase (OPM). The horizontal axis represents the ratio of the interparticle interactions to the tunneling strength, whereas the vertical axis corresponds to the ratio of the energy imbalance to the tunneling strength. The dashed yellow line shows the transition points of the effective model in the strongcoupling effective (Ising) model. The dashed red line indicates the transition as obtained from the weak-coupling expansion. The red circle shows the transition point in the balanced model at intermediate interactions. Stars label numerical results, and the blue line is an extrapolation of the phase boundaries. The labels of the critical lines give the central charge of their underlying conformal field theory. Details on the different phases and transitions between them are provided in Sec. III.

methods allow us to locate exactly certain critical points or lines and to predict topological quantum phase transitions for weak and intermediate interactions with different underlying conformal field theories (CFTs), i.e., Dirac vs Majorana CFTs, which then fit very well with numerical results based on matrix product states (MPS) [36]. We also provide suggestions for experimental observables to pinpoint these three phases.

This paper is organized as follows. In Sec. II, we introduce the standard Creutz ladder, and some previous studies are briefly accounted for which show that this model leads to a topological insulator in the BDI symmetry class [10]. Therefore, the standard Creutz ladder lies in the same symmetry class as the Su-Schrieffer-Heeger model [37], which has already been implemented in optical lattices [38]. We then introduce the imbalanced Creutz ladder, which provides an instance of a topological insulator in the AIII class (chiral unitary), which still lacks an AMO implementation. Moreover, as we argue in Sec. III, the Hubbard interactions lead to a very neat interplay of strongly correlated and topological effects. We identify the different phases of the model, together with standard and topological quantum phase transitions that connect them. Finally, in Sec. IV, we lay out a detailed proposal to implement the imbalanced Creutz-Hubbard ladder in an ultracold Fermi gas with two different internal states trapped in a standard one-dimensional optical lattice, enriched by 
intensity-shaking and Raman laser-assisted tunneling. We present our conclusions and outlook in Sec. V.

\section{IMBALANCED CREUTZ-HUBBARD LADDER}

The standard Creutz model describes a system of spinless fermions on a two-leg ladder [see Fig. 2(a)], which are created-annihilated by the fermionic operators $c_{i, \ell}^{\dagger}, c_{i, \ell}$, where $i \in\{1, \ldots, N\}$ labels the lattice sites within each leg of the ladder $\ell \in\{u, d\}$. Fermions are allowed to hop vertically along the rungs of the ladder with tunneling strength $t_{v}$ and horizontally along the legs of the ladder with a complex tunneling $t_{\ell}=t_{h}\left(e^{i \theta} \delta_{\ell, u}+e^{-i \theta} \delta_{\ell, d}\right)$, where $t_{h}$ is a tunneling strength and $\delta_{a, b}$ is the Kronecker delta. The arrangement of complex phases in the horizontal links leads to a net $2 \theta$ flux gained by a fermion hopping around a square unit cell, playing thus the role of the so-called Peierls phases of a magnetic field piercing the ladder. In addition, the kinetic part of the Hamiltonian also includes a diagonal tunneling of strength $t_{\text {diag }}$, yielding altogether

$$
\begin{aligned}
H_{C}= & -\sum_{i} \sum_{\ell}\left(t_{\ell} c_{i+1, \ell}^{\dagger} c_{i, \ell}+t_{\mathrm{diag}} c_{i+1, \ell}^{\dagger} c_{i, \bar{\ell}}\right. \\
& \left.+t_{v} c_{i, \ell}^{\dagger} c_{i, \bar{\ell}}+\text { H.c. }\right),
\end{aligned}
$$

where we use the notation $\bar{\ell}=d(\bar{\ell}=u)$ for $\ell=u(\ell=d)$.

This quadratic lattice model was put forth in Ref. [35] as a simple toy model to understand some of the key properties of higher-dimensional domain-wall fermions [39,40], which were introduced in the context of lattice gauge theories to bypass the fermion-doubling problem [41]. For periodic boundary conditions, this model leads to a couple of bands that display a pair of massive Dirac fermions with different Wilson masses $m_{0}, m_{\pi}$ at momenta $k_{D} \in$ $\{0, \pi\}$ [42]. For open boundary conditions, a pair of zeroenergy modes exponentially localized to the left or right edges of the ladder appear as one of the Wilson masses gets inverted $\left(m_{\pi}<0\right)$ when $t_{v}<2 t_{\text {diag. }}$. Considering the bulkboundary correspondence discussed in Sec. I, these edge
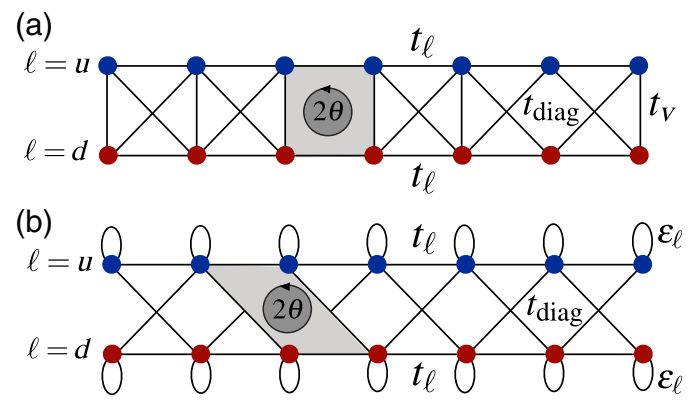

FIG. 2. Standard and imbalanced Creutz ladder. Two-leg ladder where fermions tunnel along the black links enclosing a net flux $2 \theta$ along a closed plaquete: (a) standard Creutz ladder Eq. (1) and (b) imbalanced Creutz ladder, which leads to Eq. (4) in the $\pi$-flux limit. states resemble the holographic liquid of the higherdimensional topological insulators. In fact, the change in polarization of the system can be characterized by a topological invariant [43], the so-called Zak's phase [44], such that the appearance of these zero-energy modes coincides with a nonvanishing topological invariant, and the Creutz ladder yields a symmetry-protected topological phase in this regime. As we discuss below, for $\theta=\pi / 2$, this topological phase corresponds to a BDI topological insulator.

Since the objective of this work is to study correlation effects, we now consider the simplest possible Hubbard interactions between the spinless fermions,

$$
H_{H}=\sum_{i} \sum_{\ell}\left(V_{h} n_{i, \ell} n_{i+1, \ell}+\frac{V_{v}}{2} n_{i, \ell} n_{i, \bar{\ell}}\right),
$$

where $V_{h}\left(V_{v}\right)$ are the density-density interaction strengths between fermions residing in neighboring sites along horizontal (vertical) bonds of the ladder, and we introduce the fermion number operators $n_{i, \ell}=c_{i, \ell}^{\dagger} c_{i, \ell}$.

For reasons that will become clear in the cold-atom implementation discussed in Sec. IV, in the following we deal with a variant of the Creutz Hamiltonian: (i) we substitute the vertical tunneling by an energy imbalance between the legs of the ladder $\varepsilon_{u}=\Delta \varepsilon / 2=-\varepsilon_{d}$, which changes the symmetry class of the topological insulator for $\theta=\pi / 2$ from BDI to AIII; (ii) we limit the interaction terms Eq. (2) to the anisotropic regime $V_{h}=0$; (iii) we set the amplitude of the diagonal hopping equal to the one along the legs $\left(\left|t_{\text {diag }}\right|=\left|t_{\ell}\right|=\tilde{t}\right)$; and finally (iv) we fix the phases in order to get a net $\pi$ flux through the plaquettes. The resulting Hamiltonian [see Fig. 2(b)], which we refer to as the imbalanced Creutz-Hubbard Hamiltonian, is

$$
H_{\pi \mathrm{CH}}=H_{\pi C}+V_{\mathrm{Hubb}}, \quad H_{\pi C}=H_{\mathrm{FB}}+V_{\mathrm{imb}},
$$

where we introduce the kinetic term

$$
H_{\mathrm{FB}}=\sum_{i, \ell}\left(-\tilde{t} c_{i+1, \ell}^{\dagger} c_{i, \bar{\ell}}+i s_{\ell} \tilde{t} c_{i+1, \ell}^{\dagger} c_{i, \ell}+\text { H.c. }\right),
$$

with $s_{u / d}= \pm 1$. This term leads to a pair of flat bands and a couple of zero-energy topological edge states. The remaining terms,

$$
V_{\mathrm{imb}}=\sum_{i, \ell} \frac{\Delta \varepsilon}{2} s_{\ell} n_{i, \ell}, \quad V_{\mathrm{Hubb}}=\sum_{i, \ell} \frac{V_{v}}{2} n_{i, \ell} n_{i, \bar{\ell}},
$$

contain the Hubbard interactions and the energy imbalance, which can have nontrivial effects on the flat-band physics and induce a phase transition to other nontopological phases of matter. In the following section, we present a new formalism to understand such transitions.

We are encouraged to study this imbalanced CreutzHubbard ladder for both experimental and theoretical reasons 
that we discuss in detail in the forthcoming sections. In particular, let us advance that this model shares topological properties with the original Hamiltonian Eq. (1). In particular, the Dirac fermions now occur at $k_{D} \in\{-\pi / 2, \pi / 2\}$ with different Wilson masses $m_{ \pm \pi / 2}$. Provided that $\Delta \varepsilon<$ $4 t_{h}=4 t_{d}$, the mass $m_{\pi / 2}<0$ gets inverted, and we obtain analogous topological features and exponentially localized edge states. The choice of the anisotropic regime $V_{h}=0$ is motivated by the use of ultracold fermionic atoms in optical lattices with contact interactions. Exploring also the regime of $V_{h}>0$ would require situations where the atoms have longer-range interactions.

\section{A. Previous studies on related models}

With the model under study already defined, let us comment on some relevant literature and advance some comments in relation to our results. The standard Creutz ladder Eq. (1) filled with bosons has been studied in Refs. [45,46], which includes on-site Hubbard interactions instead of the nearest-neighbor terms of Eq. (2). The focus of Refs. $[45,46]$ was the appearance of pair superfluids due to the interplay of interactions and frustration flat-band effects [47]. A useful formalism was introduced in these works, which consisted of the projection of the Hamiltonian onto the lowest-energy flat band by introducing highly localized Wannier functions. Moreover, using a unitarily equivalent formulation of the Creutz-ladder Hamiltonian introduced in Ref. [46] would lead to a spin-orbit coupled Hubbard model with staggered energy imbalance in our case Eq. (3), which might broaden the relevance of our results beyond the ladder compound of Fig. 2. The standard Creutz ladder Eq. (1) populated by spinful fermions with on-site interactions between opposite spins has been studied recently in Ref. [48], which focused on an exact Bardeen-CooperSchrieffer description for attractive Hubbard interactions. To the best of our knowledge, the standard Creutz ladder with spinless fermions has been studied previously only in Ref. [49]. Here, the emphasis was placed on an additional superconducting $s$-wave pairing, and its interplay with the repulsive Hubbard interactions, which leads to an interesting Creutz-Majorana-Hubbard ladder. The limit of vanishing pairing, which corresponds to the standard Creutz-Hubbard model with the vertical tunneling Eq. (1) instead of the imbalance Eq. (5), and leads to the aforementioned BDI topological insulator, was only touched upon briefly by numerical mean-field and density-matrix renormalization group studies. These results pointed towards the possibility of a phase with in-plane ferromagnetic order as the interactions are increased.

To the best of our knowledge, the effect of Hubbard interactions in the imbalanced Creutz ladder Eq. (3), and more generally on AIII topological insulators, remains largely unexplored. In relation to the above studies on related models, we show below that one cannot project onto a single flat band for the fermionic model, but must instead retain all flat bands and edge modes to account for correlation and topological effects accurately. In this way, we make interesting connections between the existence of topological phase transitions and the physics of quantum impurity models. Additionally, by going beyond a mean-field analysis in the strong-interaction limit of the imbalanced model Eq. (3), we show analytically that inplane ferromagnetic order also arises in our model, and moreover corresponds to the symmetry-broken phase of an orbital quantum Ising model. In addition, we illustrate that the imbalance drives such an Ising model into an orbital paramagnetic phase.

\section{TOPOLOGICAL QUANTUM PHASE TRANSITIONS}

The following sections are devoted to the construction of the phase diagram shown in Fig. 1. We start by discussing the solution of the noninteracting imbalanced Creutz ladder and the appearance of flat bands and fully localized edge states in the Hamiltonian Eq. (4) (see Sec. III A). This corresponds to the vertical axis of the phase diagram. In Sec. III B, we examine the weakly interacting regime and show that the model maps onto a pair of weakly coupled Ising chains, which can be studied through a mean-field analysis (i.e., the region in the vicinity of the vertical axis of Fig. 1). In Sec. III C, we study the opposite limit of very strong interactions (i.e., rightmost region of Fig. 1) and discuss the possible nontopological orbital magnetic phases that can arise. In Sec. III D, we explore the intermediate regime and show that the effect of the interactions and imbalance leads to edge-bulk couplings that can be mapped onto quantum impurity models. This new perspective yields a neat picture underlying the destabilization of the topological phase in favor of the orbital magnets. These different methods allow us to build an analytical prediction of the phase diagram of the model. Finally, in Sec. III E, we test numerically the above predictions and provide a detailed study of the phase diagram by means of matrix product state numerical simulations. Scattered through these sections, we also introduce comments on possible experimental tools to measure the relevant observables for the different phases of the model, which become relevant for the specific experimental cold-atom proposal we discuss in Sec. IV.

\section{A. Noninteracting limit: Flat bands and edge states}

We start by solving the kinetic part [Eq. (4)] of the $\pi$-flux Creutz-Hubbard Hamiltonian Eq. (3). For periodic boundary conditions, and after introducing the spinor $\Psi(q)=$ $\left(c_{u}(q), c_{d}(q)\right)^{t}$ for the fermion operators in momentum space $c_{\ell}(q)=\sum_{i} e^{-i q a i} c_{i, \ell} / \sqrt{N}$, one finds

$$
H_{\mathrm{FB}}=\sum_{q \in \mathrm{BZ}} \Psi^{\dagger}(q) \boldsymbol{B}(q) \cdot \boldsymbol{\sigma} \Psi(q),
$$


where $\boldsymbol{\sigma}=\left(\sigma_{x}, \sigma_{y}, \sigma_{z}\right)$ is the vector of Pauli matrices and $\boldsymbol{B}(q)=2 \tilde{t}(-\cos (q a), 0, \sin (q a))$. By direct diagonalization, one finds that the system develops two flat bands, $\varepsilon_{ \pm}:=\varepsilon_{ \pm}(q)= \pm 2 \tilde{t}$, where $q \in \mathrm{BZ}=[-\pi / a, \pi / a)$ is the quasimomentum, and the lattice constant is set to $a=1$ henceforth. The vanishing group velocity associated with these bands, $v_{g}=\partial_{q} \varepsilon_{ \pm}(q)=0$, indicates that the ground state must be insulating, regardless of the particular filling. This can be considered as a new type of insulator, namely, a flat-band insulator, which corresponds neither to the usual band insulator nor to the Mott insulators. It shares some properties with the former (i.e., no correlations) and with the latter (i.e., localized fermions), but it differs from both insulators in the large degeneracy of the ground state, except for half-filling conditions.

On top of this, the flat bands are also topological: the diagonalization of the discrete chiral symmetry $\sigma_{y}$ [s.t. $\sigma_{y} H(q) \sigma_{y}=-H(q)$, with $\left.H(q)=\boldsymbol{B}(q) \cdot \boldsymbol{\sigma}\right]$ puts the Hamiltonian Eq. (6) in a purely off-diagonal form, with elements $B_{x} \pm i B_{z}$; its complex phase gets a nontrivial winding number $\mathcal{W}=\operatorname{sgn}(\tilde{t}) \neq 0$ [50]. Equivalently, we could consider the eigenvectors $\left|\varepsilon_{ \pm}(q)\right\rangle \propto\left[\left(B_{x}+i B_{z}\right)^{1 / 2}\right.$, $\left.\pm\left(B_{x}-i B_{z}\right)^{1 / 2}\right]^{t}$ and realize that they exhibit a uniform Berry connection $\mathcal{A}_{ \pm}(q)=i\left\langle\varepsilon_{ \pm}(q)\left|\partial_{q}\right| \varepsilon_{ \pm}(q)\right\rangle=\frac{1}{2}$. The uniform Berry connection leads to a finite Zak's phase, which is defined [44] as

$$
\varphi_{\mathrm{Zak}, \pm}=\int_{\mathrm{BZ}} d q \mathcal{A}_{ \pm}(q)
$$

and equals $\varphi_{\mathrm{Zak}, \pm}=\pi$ for our topological flat bands. This Zak's phase pinpoints the topological properties of the bands, and can be connected to a macroscopic observable: the polarization of the system [43].

Interestingly enough, the Creutz ladder displays an infinite flatness parameter without requiring long-range tunnelings, as is necessary in higher-dimensional models of topological flat bands [51]. From this perspective, switching on the leg imbalance $\Delta \varepsilon>0$ in Eq. (5) leads to some curvature in the energy bands,

$$
\varepsilon_{ \pm}(q)= \pm \varepsilon(q)= \pm 2 \tilde{t} \sqrt{1+\mathfrak{f}^{-2}+2 \mathfrak{f}^{-1} \sin q},
$$

where we introduce the flatness parameter $\mathfrak{f}=4 \tilde{t} / \Delta \varepsilon$, which becomes infinite for vanishing imbalance. The presence of the imbalance also drives the system out of the BDI class and into the AIII class [10], since $B_{z}(q)=\Delta \varepsilon / 2+2 \tilde{t} \sin q$ acquires a mixed parity under $k \leftrightarrow-k$ and therefore no effective time-reversal $\left[U_{T} H(-q)^{*} U_{T}^{\dagger}=+H(q)\right]$ or particle-hole $\left[U_{C} H(-q)^{*} U_{C}^{\dagger}=-H(q)\right]$ operators can be found. Anyway, the discrete chiral symmetry is still described by $\sigma_{y}$ and the whole procedure described above can be employed. The Berry connection of the bands becomes nonuniform due to their curvature,

$$
\mathcal{A}_{ \pm}(q)=\frac{1+\mathfrak{f}^{-1} \sin q}{2\left(1+\mathfrak{f}^{-2}+2 \mathfrak{f}^{-1} \sin q\right)}
$$

which leads to the following Zak's phase: $\varphi_{\mathrm{Zak}, \pm}=$ $\pi \theta(\mathfrak{f}-1)$, where $\theta(x)$ is the Heaviside step function. Hence, the Zak's phase yields topological effects provided that the bands are sufficiently flat, i.e., $\mathfrak{f}>1$. Conversely, when $\Delta \varepsilon>4 \tilde{t}$, the curvature of the bands is large, i.e., $\mathfrak{f}<1$, and no topological phenomenon occurs. We note that this point $\Delta \varepsilon=4 \tilde{t}$ is exactly the regime where one of the fermionic Wilson masses vanishes, as mentioned in the previous section in connection to the domain-wall fermions of lattice gauge theories. This marks a quantum phase transition between the AIII topological insulator and a trivial band insulator, as shown in the vertical axis of Fig. 1 .

Regarding cold-atom experiments, we note that the Zak's phase has been measured for another paradigmatic topological insulator in 1D: the Su-Schrieffer-Heeger model of polyacetilene [37]. In this noninteracting limit, the Zak's phase is a single-particle property of the topological bands, and can be thus measured by a Ramsey interferometric protocol that exploits Bloch oscillations of single-particle initial states [38]. This measurement scheme has been generalized to other topological insulators [52], and can also be applied to the cold-atom implementation of the Creutz ladder considered in Sec. IV.

To have an alternative view on these topological features, let us introduce the so-called Aharonov-Bohm cages $(\mathrm{ABC})$ [53], which will also become very useful once interactions are switched on. In the $\pi$-flux Creutz ladder Eq. (4), the fermions cannot tunnel two sites apart due to the Aharonov-Bohm effect [35] [see Fig. 3(a)]. One can thus find single-particle eigenstates strictly localized in cages formed by simple square plaquettes [see Fig. 3(c)]. In second quantization, such Aharonov-Bohm cages with energies $\varepsilon_{ \pm}= \pm 2 \tilde{t}$ are

$$
\begin{aligned}
|+2 \tilde{t}\rangle_{i} & =w_{i,+}^{\dagger}|0\rangle, \\
w_{i,+}^{\dagger} & =\frac{1}{2}\left(i c_{i, u}^{\dagger}+c_{i, d}^{\dagger}-c_{i+1, u}^{\dagger}-i c_{i+1, d}^{\dagger}\right), \\
|-2 \tilde{t}\rangle_{i} & =w_{i,-}^{\dagger}|0\rangle, \\
w_{i,-}^{\dagger} & =\frac{1}{2}\left(i c_{i, u}^{\dagger}+c_{i, d}^{\dagger}+c_{i+1, u}^{\dagger}+i c_{i+1, d}^{\dagger}\right),
\end{aligned}
$$

with $i \in\{1, \ldots, N\}$ for periodic boundary conditions, where one identifies $c_{N+1, \ell}=c_{1, \ell}$. Conversely, for open boundary conditions, these square $\mathrm{ABC}$ can be defined for only $i \in\{1, \ldots, N-1\}$, and simple counting shows that there are only $2(N-1)$ possible states that can be accommodated in such flat bands. The two missing states are zero-energy modes, $\varepsilon_{l}=\varepsilon_{r}=0$, fully localized at the boundaries 
(a)

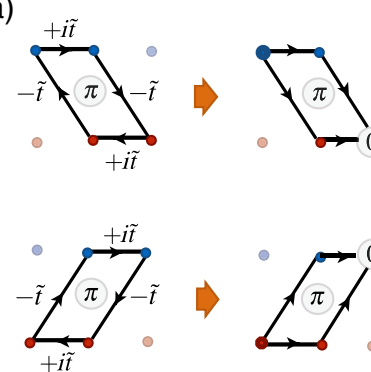

(b)

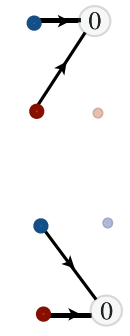

(c)

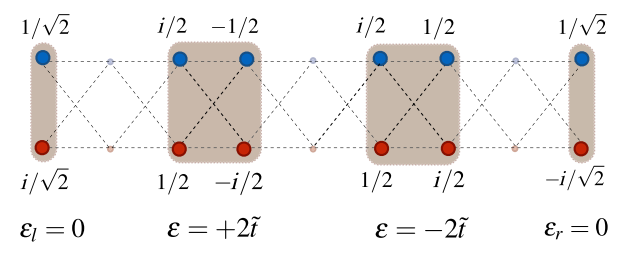

FIG. 3. Aharonov-Bohm cages in the Creutz ladder. (a) Considering the tunneling paths in the Creutz ladder Eq. (4), one can identify two types of rhombic plaquettes that enclose a synthetic $\pi$ flux (left). Therefore, a particle trying to tunnel two sites apart (middle) will be subjected to a destructive Aharonov-Bohm interference that forbids this process. Accordingly, particles are confined to the so-called Aharonov-Bohm cages and cannot spread through the entire lattice. These cages correspond to square plaquettes, except at the edges (b), where destructive interference can also be found for a particle trying to tunnel one site apart (right). (c) Aharonov-Bohm cages with the relative amplitudes for a single-particle state in two possible flat bands and in the two possible zero-energy edge modes.

$$
\begin{array}{ll}
|0\rangle_{L}=l^{\dagger}|0\rangle, & l^{\dagger}=\frac{1}{\sqrt{2}}\left(c_{1, u}^{\dagger}+i c_{1, d}^{\dagger}\right), \\
|0\rangle_{R}=r^{\dagger}|0\rangle, & r^{\dagger}=\frac{1}{\sqrt{2}}\left(c_{N, u}^{\dagger}-i c_{N, d}^{\dagger}\right) .
\end{array}
$$

For these particular weights, the fermions cannot tunnel one site apart due to the Aharonov-Bohm effect [see Fig. 3(a)], and are thus localized within a boundary $\mathrm{ABC}$ [see Fig. 3(b)], which corresponds to an edge state within the bulk-edge correspondence of the topological insulator.

We can finally express the $\pi$-flux Creutz Hamiltonian Eq. (3) for open boundary conditions as

$$
H_{\mathrm{FB}}=\sum_{i=1}^{N-1} \sum_{\alpha= \pm} \varepsilon_{\alpha} w_{i, \alpha}^{\dagger} w_{i, \alpha}+\sum_{\eta=l, r} \varepsilon_{\eta} \eta^{\dagger} \eta .
$$

Although generic fillings can lead to a variety of interesting phases in the presence of interactions, potentially connected to fractional topological insulators [51], we are concerned in this work with only half filling, i.e., $N$ fermions, where the ground state of Eq. (12) is twofold degenerate,

$$
\begin{aligned}
\left|\varepsilon_{g}, L\right\rangle_{\mathrm{NI}} & =l^{\dagger} w_{1,-}^{\dagger} w_{2,-}^{\dagger} \cdots w_{N-1,-}^{\dagger}|0\rangle, \\
\left|\varepsilon_{g}, R\right\rangle_{\mathrm{NI}} & =r^{\dagger} w_{1,-}^{\dagger} w_{2,-}^{\dagger} \cdots w_{N-1,-}^{\dagger}|0\rangle,
\end{aligned}
$$

and the ground-state energy is

$$
\varepsilon_{g, L}=\varepsilon_{g, R}=-2 \tilde{t}(N-1) .
$$

We thus see that the ground-state degeneracy corresponds to the two possible choices in populating either of the zeroenergy edge modes, and it is related to the topology of the ladder (i.e., ring versus line with open edges).

The effects of the leg imbalance $\Delta \varepsilon>0$ in Eq. (5) can be understood from this edge perspective by writing

$$
\begin{aligned}
V_{\mathrm{imb}}= & \sum_{i=2}^{N-1} t_{\mathrm{imb}}\left(w_{i-1,+}^{\dagger}-w_{i-1,-}^{\dagger}\right)\left(w_{i,+}+w_{i,-}\right) \\
& +\sum_{\alpha= \pm} \sqrt{2} t_{\mathrm{imb}}\left(-l^{\dagger} w_{1, \alpha}-i \alpha r^{\dagger} w_{N-1, \alpha}\right)+\text { H.c. }
\end{aligned}
$$

where $t_{\text {imb }}=-i \Delta \varepsilon / 4$ is an effective tunneling induced by the imbalance, and has two relevant effects. The first line describes the hopping of fermions in neighboring $\mathrm{ABC}$, which leads to the aforementioned curvature of the bulk energy bands Eq. (8). The second line represents the hopping between the topological edge modes and the bulk ABC. As discussed in more detail in Sec. III D, both terms conspire to induce a broadening of the edge modes in the regime $4 \tilde{t} \leq \Delta \varepsilon$, which is the regime where the topological Zak's phase Eq. (7) vanishes, signaling an imbalanceinduced topological phase transition. We also note that, precisely at the point $4 \tilde{t}=\Delta \varepsilon$, the bulk bands become $\varepsilon_{ \pm}(q)= \pm 2 \tilde{t}(1+\sin q)$, and the gap vanishes exactly at $q=-\pi / 2$, which coincides with the momentum of the massless Wilson fermion.

Regarding cold atoms, previous experiments on synthetic two-leg ladders subjected to artificial gauge fluxes, where the diagonal interleg tunneling in Eq. (1) is substituted by a vertical one, have measured nonvanishing chiral currents circulating in opposite directions along each leg of the ladder [54]. These states are connected to the edge states of the Hofstadter model as the number of legs is increased [54,55], but the bulk and edges coincide in the limiting case of the two-leg ladder. The situation is different for the two-leg Creutz ladder, as the edge states are not extended along the legs of the ladder but exponentially localized to the left- and rightmost rungs Eq. (11). We note that observing the particular localization in Eq. (11) would require implementing a box confining potential, which has already been achieved in several cold-atom experiments for bosonic gases [56]. However, we note that for milder confining potentials, 
one expects that the edge states will remain to be confined in the boundaries of the system, provided that the confining potential increases at least quadratically with the distance to the center of the trap [57].

According to the above discussion, no additional legs would be required to identify the difference between edge and bulk excitations in the Creutz ladder, such that detecting the presence of zero-energy modes localized at the edges would be a proof of the topological nature of the phase. Using a pair of laser beams with a frequency difference that can be scanned within the edge-bulk gap $\left|\omega_{L, 1}-\omega_{L, 2}\right|<2 \tilde{t}$ and a spatial profile that can be localized to a few sites from the left- and rightmost rungs of the ladder, the associated Bragg signal due to Raman excitations can detect the presence of these localized zero modes, provided that the atomic gas is confined in a box potential. This method is analogous to the situation in higher-dimensional topological insulators [58], where additional angular momentum of the laser beams can be exploited to probe the chirality of the edge modes and improve the measurement resolution [57]. For milder confinement potentials, nontopological states might also happen to be localized within the edges of the system [58]. In Ref. [57], the authors show that the Bragg scheme can be adapted to distinguish the chirality of the localized edge states of a higher-dimensional topological insulator, and thus differentiate them from the nontopological states, probing in this way the topological nature of the phase. It would be interesting to explore analog techniques to differentiate the edge states Eq. (11) of the Creutz ladder from spurious states that would arise if no box potential is implemented.

\section{B. Weak interactions: Quantum Ising ladder}

Let us now address the fate of this topological phase as the Hubbard repulsion is switched on. We start by exploring the weakly interacting regime $\tilde{t} \gg V_{v}$, and by noting that the two representations of the noninteracting imbalanced model $H_{\pi C}=H_{\pi C}(\tilde{t}, \Delta \varepsilon)$ Eq. (3), both in the original [Eqs. (1) and (2)] and in the plaquette [Eqs. (12) and (15)] bases, are dual to each other. In particular, the mapping between spinors,

$$
\Psi_{i}=\left(c_{i, u}, c_{i, d}\right)^{t} \rightarrow W_{i}=e^{-i(\pi / 4) \sigma_{i}^{z}}\left(w_{i,+}, w_{i,-}\right)^{t},
$$

induces a duality transformation $H_{\pi C}(\tilde{t}, \Delta \varepsilon) \rightarrow(\Delta \varepsilon / 4 \tilde{t}) \times$ $H_{\pi C}\left[\tilde{t},\left(16 \tilde{t}^{2} / \Delta \varepsilon\right)\right]$ with a self-dual point $\Delta \varepsilon=(4 \tilde{t})^{2} / \Delta \varepsilon$ corresponding to the previous critical point $\Delta \varepsilon=4 \tilde{t}$.

Such a duality is reminiscent of the one occurring in the QIM [59] and suggests a possible equivalence between both models. Indeed, a formal equivalence is found by introducing the following rung operators:

$$
\begin{aligned}
& r_{j, 1}=\frac{i^{j}}{\sqrt{2}}\left[i c_{j, u}+(-1)^{j} c_{j, d}^{\dagger}\right], \\
& r_{j, 2}=\frac{i^{j}}{\sqrt{2}}\left[c_{j, u}+i(-1)^{j} c_{j, d}^{\dagger}\right] .
\end{aligned}
$$

Under this canonical transformation, the Hamiltonian is transformed onto

$$
\begin{aligned}
H_{\pi C}= & -\tilde{t} \sum_{j} \sum_{n=1,2}\left(r_{j, n}^{\dagger} r_{j+1, n}+r_{j, n}^{\dagger} r_{j+1, n}^{\dagger}+\text { H.c. }\right) \\
& +\frac{\Delta \varepsilon}{4} \sum_{j} \sum_{n=1,2}\left(2 r_{j, n}^{\dagger} r_{j, n}-1\right) .
\end{aligned}
$$

In this particle-hole rung basis Eq. (17), we identify two independent subsystems which no longer display particlenumber conservation, but instead have parity conservation. A Jordan-Wigner transformation [60], namely,

$r_{j, n}^{\dagger}=\prod_{i<j}\left(-\sigma_{i, n}^{z}\right) \sigma_{j, n}^{+}=\left(r_{j, n}\right)^{\dagger}, \quad r_{j, n}^{\dagger} r_{j, n}=\frac{1}{2} \sigma_{j, n}^{z}+\frac{1}{2}$,

reveals the Ising nature of the two subsystems, and leads to a Hamiltonian that can be understood as a two-leg quantum Ising ladder:

$$
H_{\pi C}=\sum_{j} \sum_{n=1,2}\left(-\tilde{t} \sigma_{j, n}^{x} \sigma_{j+1, n}^{x}+\frac{\Delta \varepsilon}{4} \sigma_{j, n}^{z}\right)
$$

For open boundary conditions, this description allows an alternative interpretation of the edge-state behavior by writing the Ising model as a Kitaev-Majorana chain [8]. The ferromagnetic regime is associated with two uncoupled Majorana zero-energy modes on the opposing edges of the system for each of the legs of the Ising ladder. Combining the two free Majoranas on either edge of the Ising ladder then yields the local fermionic edge modes defined in Eq. (11). This contrasts the case of a single Majorana chain, where the fermionic zero mode is highly nonlocal since it can only be built from the Majoranas at the opposite edges of the chain.

Let us turn on the interactions and express the weak Hubbard repulsion in terms of these rung operators:

$$
\begin{aligned}
V_{\mathrm{Hubb}}= & \frac{V_{v}}{4} \sum_{j}\left(i r_{j, 1}^{\dagger} r_{j, 2}+\text { H.c. }\right) \\
& -\frac{V_{v}}{4} \sum_{j}\left[\left(1-2 r_{j, 1}^{\dagger} r_{j, 1}\right)\left(1-2 r_{j, 2}^{\dagger} r_{j, 2}\right)-1\right] .
\end{aligned}
$$

These terms introduce a coupling between the two legs of the quantum Ising ladder. If we restrict to half filling in the original model, the tunneling term vanishes, and we are left 
with the quartic interactions. These in turn can be expressed in terms of spin-spin interactions that couple the two legs of the ladder:

$$
V_{\mathrm{Hubb}}=-\frac{V_{v}}{4} \sum_{j} \sigma_{j, 1}^{z} \sigma_{j, 2}^{z}+\text { const. }
$$

For weak interactions, $V_{v} \ll \Delta \varepsilon, \tilde{t}$, we can treat the influence of one Ising chain on the remaining one with a mean-field approximation,

$$
H_{\pi \mathrm{CH}} \approx \sum_{j, n}\left(-\tilde{t} \sigma_{j, n}^{x} \sigma_{j+1, n}^{x}+\frac{\Delta \varepsilon-V_{v} m_{\bar{n}}\left(\Delta \varepsilon, V_{v}, \tilde{t}\right)}{4} \sigma_{j, n}^{z}\right),
$$

where we introduce the transverse magnetization $m_{\bar{n}}\left(\Delta \varepsilon, V_{v}, \tilde{t}\right)=\left\langle\sigma_{j, \bar{n}}^{z}\right\rangle$ for each leg of the ladder, and $\bar{n}=2,1$ for $n=1,2$. We thus observe a renormalization of the imbalance parameter that controls the transverse field of the Ising model, and thus leads to a shift of the critical point as the interaction strength $V_{v}$ increases.

Accordingly, the topological phase of Sec. III A survives in a finite region of parameter space as the interactions are switched on. We find this region by solving the self-consistency mean-field equations by iterating

$$
m_{n}\left(\Delta \varepsilon, V_{v}, \tilde{t}\right)=M\left(\frac{\Delta \varepsilon-V_{v} m_{\bar{n}}\left(\Delta \varepsilon, V_{v}, \tilde{t}\right)}{4 \tilde{t}}\right)
$$

to convergence. In this equation, we use the exact expression of the ground-state transverse magnetization in the QIM [61], namely,

$$
M(\alpha)= \begin{cases}\frac{2\left(1-\alpha^{2}\right)}{\pi \alpha}\left[\Pi\left(\alpha^{2}, \alpha\right)-K(\alpha)\right] & \text { for } \alpha<1 \\ \frac{2\left(\alpha^{2}-1\right)}{\pi \alpha^{2}} \Pi\left(1 / \alpha^{2}, 1 / \alpha\right) & \text { for } \alpha>1\end{cases}
$$

where we introduce the complete elliptic integrals of the first and third kind, namely,

$$
\begin{gathered}
K(k)=\int_{0}^{\pi / 2} d \theta \frac{1}{\sqrt{1-k^{2} \sin ^{2} \theta}}, \\
\Pi(n, k)=\int_{0}^{\pi / 2} d \theta \frac{1}{\left(1+n \sin ^{2} \theta\right) \sqrt{1-k^{2} \sin ^{2} \theta}} .
\end{gathered}
$$

The self-consistent mean-field approximation reproduces very precisely the numerical results for the density imbalance of the Creutz ladder:

$$
\Delta n=\frac{1}{N} \sum_{i}\left(c_{i, u}^{\dagger} c_{i, u}-c_{i, d}^{\dagger} c_{i, d}\right)=\frac{1}{2 N} \sum_{j} \sum_{n} \sigma_{j, n}^{z} .
$$

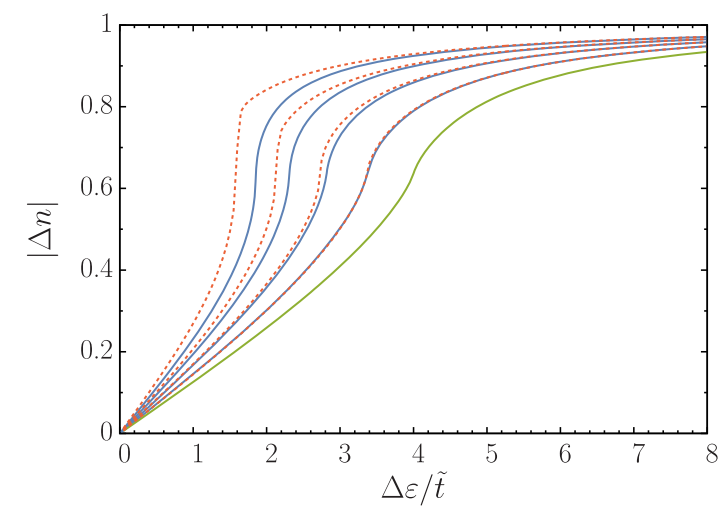

FIG. 4. Exact density imbalance and mean-field magnetizations. In blue solid lines, we present the ground-state density imbalance $\langle\Delta n\rangle$ for systems with $V_{v} / \tilde{t}=1,2,3,4$ (from bottom to top) as determined numerically by means of a matrix product state algorithm for a ladder with $L=256$ sites. In red dashed lines, we show the mean-field magnetizations $m_{1}\left(\Delta \varepsilon, V_{v}, \tilde{t}\right)=$ $m_{2}\left(\Delta \varepsilon, V_{v}, \tilde{t}\right)$ of the coupled Ising chains. The agreement $\langle\Delta n\rangle \approx \frac{1}{2}\left[m_{1}\left(\Delta \varepsilon, V_{v}, \tilde{t}\right)+m_{2}\left(\Delta \varepsilon, V_{v}, \tilde{t}\right)\right]$ is reasonable even for considerably large interaction strengths. The green curve shows as a reference the magnetization of the noninteracting model $M(\Delta \varepsilon / 4 \tilde{t})$.

In Fig. 4, we compare the ground-state density imbalance $\langle\Delta n\rangle$ with the mean-field transverse magnetization $m_{1}\left(\Delta \varepsilon, V_{v}, \tilde{t}\right)=m_{2}\left(\Delta \varepsilon, V_{v}, \tilde{t}\right)$, and find a very good agreement even for considerable interactions $V_{v} \sim \tilde{t}$.

If we solve the self-consistency equation to first order in $V_{v} / \tilde{t}$, we find that the critical point $\Delta \varepsilon / \tilde{t}=4$ flows towards smaller values of the imbalance as the interactions increase:

$$
\frac{\Delta \varepsilon}{\tilde{t}}=4-\frac{2}{\pi} \frac{V_{v}}{\tilde{t}}+\mathcal{O}\left(\frac{V_{v}^{2}}{\tilde{t}^{2}}\right)
$$

This weak-coupling expansion defines a critical line in parameter space that separates the topological and nontopological phases and agrees well with our numerical findings for the phase diagram of the model, as discussed below (see the red dashed line in Fig. 1).

Regarding the cold-atom experiment, we note that the definition of the Zak phase Eq. (7), which could be used to probe the topological nature of the phase via interferometric methods [52], is valid only in the noninteracting limit. For weak interactions, the single-particle excitations will be described instead by quasiparticles, and the interferometric protocol to measure a many-body Zak's phase can be obtained by coupling additional impurities to the interacting Fermi gas [62]. We also note that the method to detect the zero-energy edge modes based on Bragg scattering [58] should also hold in the interacting regime. Finally, we advance that a measurement of the leg imbalance density Eq. (28) could also be used to experimentally test the validity of our prediction of the critical line Eq. (29) (see 
Sec. III E). Concerning the mapping in Eq. (78), this leg imbalance can be inferred from spin-resolved density measurements obtained by optical imaging, either in situ (dispersive imaging) or after a time-of-flight expansion (absorption imaging). Exploiting light polarization and selection rules for the particular internal states of the atoms, one could measure separately the density of each of the two components, and even spin structure factors [63] that give access to the leg-imbalance susceptibility. Note that this susceptibility can be used to extract the position of the critical line (see top panel of Fig. 6). Regarding in situ absorption imaging, spin-selective density measurements and correlations can also be accomplished [64]. As alternative possibilities, the spin structure factor could be measured in a nondemolition manner by exploiting a quantum Faraday effect [65], while spin-resolved density measurements can be achieved by using recent quantum gas microscopes (see, e.g., Ref. [66]).

\section{Strong interactions: Orbital Ising ferromagnet}

In this section, we explore the opposite limit of the Creutz-Hubbard ladder [Eq. (3)], namely, the strongly interacting regime $\tilde{t} \ll V_{v}$. In the limit $\tilde{t}=0$, the ground state of the Creutz-Hubbard Hamiltonian corresponds to a Mott insulator where the $N$ fermions are distributed in the ladder avoiding simultaneous occupancies of two sites within the same rung.

By switching on the tunneling $\tilde{t} \ll V_{v}$, an orbital analog of the well-known superexchange [67-70] takes place, which reduces the energy of the ground state by allowing for processes where a rung of the ladder is virtually occupied by two fermions. Instead of the usual Heisenberg model associated to the strongly interacting Hubbard model [67], a different spin model arises in the present case due to the combination of intra- and interleg tunnelings. To second order of perturbation theory [71], and considering the half-filling regime, the relevant Hamiltonian describing the low-energy physics corresponds to an orbital quantum Ising model, namely,

$$
\mathcal{P}_{r} H_{\pi \mathrm{CH}} \mathcal{P}_{r}=\frac{1}{4} J N+J \sum_{i} T_{i}^{y} T_{i+1}^{y}+\Delta \varepsilon \sum_{i} T_{i}^{z},
$$

where the superexchange coupling is $J=-8 \tilde{t}^{2} / V_{v}$, and the leg imbalance $\Delta \varepsilon$ plays the role of an effective transverse field. The above spin operators are defined as the orbital analogue of the usual spin operators for electrons:

$$
\begin{aligned}
T_{i}^{y} & =\frac{1}{2}\left(-i c_{i, u}^{\dagger} c_{i, d}+i c_{i, d}^{\dagger} c_{i, u}\right), \\
T_{i}^{z} & =\frac{1}{2}\left(c_{i, u}^{\dagger} c_{i, u}-c_{i, d}^{\dagger} c_{i, d}\right) .
\end{aligned}
$$

Finally, $\mathcal{P}_{r}=\Pi_{i}\left(1-n_{i, u} n_{i, d}\right)$ is a Gutzwiller projector onto the subspace of singly occupied vertical rungs.
The 1D quantum Ising model can be exactly solved [72] by introducing a Jordan-Wigner transformation [60], followed by a fermionic Bogoliubov transformation [73]. In comparison with the noninteracting ground state Eq. (13), which displays a topological twofold degeneracy, the strongly interacting ground state for $\Delta \varepsilon<|J| / 2$ has a nontopological degeneracy related to the $\mathbb{Z}_{2}$ symmetry of the Ising model. In this regime, the ground state develops long-range order as a consequence of spontaneous symmetry breaking:

$$
\lim _{r \rightarrow \infty}\left\langle T_{i}^{y} T_{i+r}^{y}\right\rangle_{\mathrm{SI}}=\frac{1}{4}\left(1-h^{2}\right)^{1 / 4},
$$

where $h=2 \Delta \varepsilon /|J|<1$. This defines a critical line,

$$
\frac{\Delta \varepsilon}{\tilde{t}}=\frac{4 \tilde{t}}{V_{v}}
$$

that separates the phase of long-range order, i.e., an orbital ferromagnet (OFM), from the disordered phase, i.e., an orbital paramagnet (OPM), and is depicted by a yellow dashed line in Fig. 1. As the leg imbalance is increased above a critical value $\left.(\Delta \varepsilon / \tilde{t})\right|_{c}=\left(4 \tilde{t} / V_{v}\right)$, a standard quantum phase transition occurs between the long-rangeordered Ising ferromagnet and a disordered orbital paramagnet, where all fermions tend to occupy the lower leg of the ladder. We note that this transition is not of a topological origin, as it can be understood by a local order parameter: the orbital magnetization $\left\langle T_{i}^{y} T_{i+r}^{y}\right\rangle_{\mathrm{SI}} \rightarrow m_{y}^{2}$.

We also note that the long-range ferromagnetic order is totally absent in the noninteracting topological ground state Eq. (13), where one finds

$$
\left\langle T_{i}^{y} T_{i+r}^{y}\right\rangle_{\mathrm{NI}}=0 .
$$

According to this discussion, it is clear that one cannot connect the noninteracting topological and strongly interacting ferromagnetic phases adiabatically. Therefore, there should be an interaction-induced topological quantum phase transition between the symmetry-protected topological phase and a state with magneto-orbital long-range order, for intermediate interactions $V_{v} / \tilde{t}$. Our analytical treatment of the strongly interacting regime also points to the possible origin of in-plane ferromagnetic order in the CreutzHubbard ladder with vertical tunnelings Eq. (1) instead of the imbalance, as recently found through a mean-field and numerical analysis [49]. Similar topological quantum phase transitions to magnetically ordered phases have also been found numerically in higher-dimensional models [16].

Concerning the cold-atom realization, we note that the orbital spin operators Eq. (31) developing the long-range order Eq. (32) in the orbital ferromagnet correspond to the standard spin operators for the two internal states of the atoms Eq. (78). Therefore, the magnetic correlations can be 
inferred from the spin structure factor, which can be measured, for instance, via Bragg scattering by playing with the polarization of the laser beams [63]. This can allow us to test experimentally the validity of the critical line Eq. (33).

\section{Intermediate interactions: Extended Hubbard models and quantum impurity physics}

In this section, we elaborate on a theory that allows us to predict this interaction-induced topological quantum phase transition by making an interesting connection to the physics of quantum impurity models. We start by expressing the Hubbard part of the Hamiltonian Eq. (5) in the basis of the bulk Eq. (10) and edge Eq. (11) Aharonov-Bohm cages. A similar step has been performed by rewriting the bosonic Creutz-Hubbard model in terms of the bulk negative energy $\mathrm{ABC}[45,46]$. This corresponds to a projection onto the low-energy flat band, which is justified for $\tilde{t} \gg V_{v}$, and is also customary in the theory of fractional topological insulators [51]. In our case, however, it is crucial to retain the positive- and zero-energy $\mathrm{ABC}$ to capture the topological phases and the possible quantum phase transitions that take place in the regime $\tilde{t} \sim V_{v}$.

In this basis, we find an extended Hubbard model,

$$
V_{\text {Hubb }}=V_{\mathrm{nn}}+V_{\mathrm{pt}}+V_{\mathrm{dt}},
$$

with a nearest-neighbor interaction between fermions confined in adjacent $\mathrm{ABC}$,

$V_{\mathrm{nn}}=\frac{V_{v}}{2} \sum_{\alpha= \pm}\left(n_{l} n_{1, \alpha}+n_{r} n_{N-1, \alpha}\right)+\frac{V_{v}}{4} \sum_{i=2}^{N-1} \sum_{\alpha, \beta= \pm} n_{i-1, \alpha} n_{i, \beta}$,

where the $\mathrm{ABC}$ number operators are $n_{i, \alpha}=w_{i, \alpha}^{\dagger} w_{i, \alpha}$ for $\alpha \in\{+,-\}$ and $n_{\eta}=\eta^{\dagger} \eta$ for $\eta \in\{\ell, r\}$. This effective repulsion between the fermions can be depicted in a new ladder scheme, where the legs of the ladder correspond to the positive or negative energy flat bands, and the sites correspond to the labels of the different bulk cages, except at the boundaries of the ladder, where the sites correspond to the edge cages (see Fig. 5). All the possible interactions, including the ones at the edges, are represented by wavy lines. We also include the nearest-neighbor intra- and interleg tunneling, which arises from interpreting the imbalance Eq. (15) within this virtual ladder.

In addition to this effective tunneling and repulsion, we also find correlated tunneling processes of two types. We obtain a pair-tunneling Hamiltonian,

$V_{\mathrm{pt}}=\tilde{J} \sum_{i=2}^{N-1}\left[w_{i-1,+}^{\dagger} w_{i-1,-}\left(w_{i,-}^{\dagger} w_{i,+}+w_{i,+}^{\dagger} w_{i,-}\right)\right]+$ H.c.,

where $\tilde{J}=-V_{v} / 4$. The first term in the parentheses describes how fermions confined in adjacent $A B C$ of opposite legs tunnel simultaneously to empty neighboring cages, and can be understood as the anticorrelated pair tunneling of Fig. 5. The second term in parentheses describes how fermions confined in adjacent AharonovBohm cages on the same leg tunnel simultaneously to empty neighboring cages, and can be understood as the correlated pair tunneling of Fig. 5. In addition, we also obtain an interleg density-dependent tunneling,

$$
\begin{aligned}
V_{\mathrm{dt}}= & T_{d} \sum_{i=2}^{N-1}\left(n_{i-1,+}+n_{i-1,-}-n_{i+1,+}-n_{i+1,-}\right) w_{i,+}^{\dagger} w_{i,-} \\
& +2 T_{d}\left(n_{l} w_{1,+}^{\dagger} w_{1,-}-n_{r} w_{N-1,+}^{\dagger} w_{N-1,-}\right)+\text { H.c., }
\end{aligned}
$$

where $T_{d}=V_{v} / 4$ is the tunneling strength. The first line describes an interleg tunneling within the bulk of the ladder that depends on the density difference of the neighboring

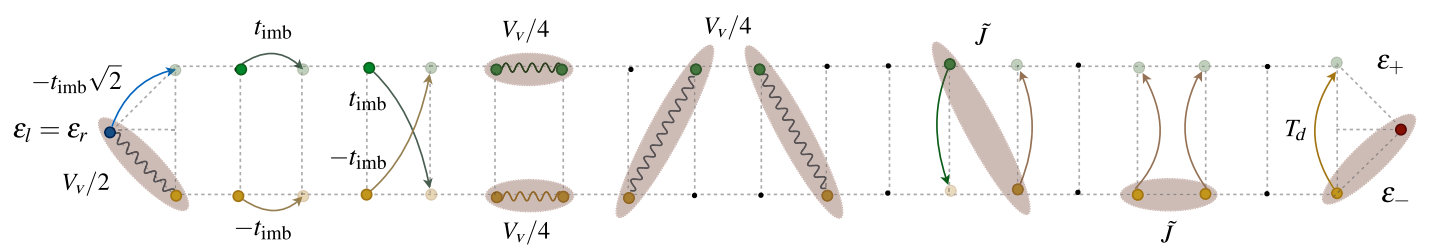

FIG. 5. Nonstandard Hubbard terms in the Aharonov-Bohm cage basis. Virtual ladder structure to represent the relevant processes in the Creutz-Hubbard ladder. The two legs of the ladder correspond to the two possible flat bands at energies $\varepsilon_{ \pm}= \pm 2 \tilde{t}$, with sites representing the bulk Aharonov-Bohm cages Eq. (10). The two boundary sites represent the two zero-energy modes $\varepsilon_{l}=\varepsilon_{r}=0$ and their corresponding edge cages Eq. (11). The imbalance of the original Creutz-Hubbard ladder Eq. (15) leads to standard intra- and interleg tunneling, but also to edge-bulk tunneling processes represented by curved arrows and with strengths proportional to $t_{\text {imb }}$. The Hubbard interaction Eq. (35) can be decomposed into nearest-neighbor interactions of strength proportional to $V_{v}$ that are represented by wavy lines and involve two adjacent bulk cages in any possible leg, or adjacent edge-bulk ones. Nonstandard Hubbard terms involving a pair tunneling also arise, where pairs of fermions tunnel simultaneously either from the same leg (correlated tunneling) or from opposite legs (anticorrelated tunneling) of the ladder with amplitude $\tilde{J}$. Finally, we also describe a density-dependent interleg tunneling of amplitude amplitude $T_{d}$, where the fermion tunneling depends on the density of fermions populating the Aharonov-Bohm cage at the neighboring edge. 
Aharonov-Bohm cages, and is negligible for a ground state with translationally invariant bulk properties. On the other hand, the second line describes interleg tunnelings that occur at the boundaries of the Creutz-Hubbard ladder, and depend on the density of the edge ABC (see Fig. 5). These terms are not negligible for translational-invariant bulks and will play a key role in the topological phase transitions of the model.

As shown in this section, the flat-band physics of the Creutz-Hubbard ladder provides an alternative route to the physics of nonstandard Hubbard models, which typically arise in optical lattices as a consequence of dipolar interactions or higher orbitals [74]. In the present case, these nonstandard terms arise due to the interplay of interactions and the flatness of the bands, which yield

$$
H_{\pi \mathrm{CH}}=H_{\mathrm{FB}}+V_{\mathrm{imb}}+V_{\mathrm{nn}}+V_{\mathrm{pt}}+V_{\mathrm{dt}},
$$

with the terms introduced in Eqs. (12), (15), and (36)-(38). At first sight, this formulation leads to a Hamiltonian [Eq. (39)] that seems more complicated than the original one [Eq. (3)]. However, as we show below, it is an ideal starting point to calculate an effective boundary theory for the topological edge states, based on which one can understand possible topological phase transitions as the imbalance and/or interactions are increased.

\section{Bulk-mediated broadening and edge-to-edge tunneling}

As a warm-up to the discussion of the model for intermediate interactions, let us discuss how to derive an effective boundary theory in the noninteracting limit $V_{v}=0$. In Sec. III A, we have already discussed how the imbalance Eq. (15) may induce a quantum phase transition at $\left.(\Delta \varepsilon / \tilde{t})\right|_{c}=4$ that can be understood through a topological invariant Eq. (7) of the bulk bands. We now discuss how an effective boundary theory, derived via the above nonstandard Hubbard Hamiltonian Eq. (39), yields an alternative description of such a phase transition.

The Hamiltonian Eq. (3) can be rearranged as follows, $H_{\pi C}=H_{\text {edge }}+H_{\text {bulk }}+H_{b-e}$, where

$$
H_{\text {edge }}=\varepsilon_{l} l^{\dagger} l+\varepsilon_{r} r^{\dagger} r
$$

stands for the Hamiltonian of the zero-energy edge modes. The bulk part of the Hamiltonian can be expressed as follows:

$$
\begin{aligned}
H_{\mathrm{bulk}}= & \sum_{i, \alpha} \varepsilon_{\alpha} w_{i, \alpha}^{\dagger} w_{i, \alpha} \\
& +\sum_{i=2}^{N-1} t_{\mathrm{imb}}\left(w_{i-1,+}^{\dagger}-w_{i-1,-}^{\dagger}\right)\left(w_{i,+}+w_{i,-}\right),
\end{aligned}
$$

which is readily diagonalized by a Fourier transform considering periodic boundary conditions $w_{N, \alpha}=w_{1, \alpha}$.
This leads to the energy bands in Eq. (8), where the quasimomentum lies in the Brillouin zone $q \in(-\pi, \pi]$, and there is a total of $2 N$ single-particle modes. To approximately solve the two-leg Creutz ladder with open boundary conditions, we build on the solution of an open single chain. We note that the bands in our ladder fulfill $\varepsilon_{\alpha}[(\pi / 2)+q]=\varepsilon_{\alpha}[(\pi / 2)-q]$, which allows us to combine modes with momenta $(\pi / 2) \pm q$ to construct $\mathrm{ABC}$ momentum operators via standing waves that respect the open boundary conditions of a finite ladder $w_{N, \alpha}=w_{0, \alpha}=0$. Note that the $-q$ solutions of the problem with periodic boundary conditions are implicitly considered in the $+q$ solutions. Together with the fact that the $q=0, \pi$ modes of the periodic solution yield trivial standing waves, this forces us to enlarge the number of sites in the problem with periodic boundary conditions $N-1 \rightarrow 2 N$, such that the allowed momenta become $q_{n}=(2 \pi / N-1) n \rightarrow q_{n}=(\pi / N) n$. The transformation that approximately diagonalizes the bulk Hamiltonian Eq. (41) is

$$
\left(\begin{array}{l}
w_{+}\left(q_{n}\right) \\
w_{-}\left(q_{n}\right)
\end{array}\right)=\sqrt{\frac{2}{N}} \sum_{j=1}^{N-1} e^{-i(\pi / 2) j} \sin \left(q_{n} j\right)\left(\begin{array}{c}
w_{j,+} \\
w_{j,-}
\end{array}\right) .
$$

Using these operators, the bulk Hamiltonian for the ladder with open ends approximately becomes

$$
H_{\text {bulk }} \approx \sum_{n=1}^{N-1} \Psi^{\dagger}\left(q_{n}\right) \boldsymbol{B}\left(q_{n}\right) \cdot \boldsymbol{\sigma} \Psi\left(q_{n}\right),
$$

where $\boldsymbol{B}\left(q_{n}\right)=\left[0,(\Delta \varepsilon / 2) \cos q_{n}, 2 \tilde{t}+(\Delta \varepsilon / 2) \sin q_{n}\right]$, and we introduce new spinors $\Psi\left(q_{n}\right)=\left(w_{+}\left(q_{n}\right), w_{-}\left(q_{n}\right)\right)^{t}$. Diagonalizing this Hamiltonian leads to the curved energy bands Eq. (8), such that $H_{\text {bulk }}=\sum_{n, \alpha} \alpha \varepsilon\left(q_{n}\right) \gamma_{\alpha}^{\dagger}\left(q_{n}\right) \gamma_{\alpha}\left(q_{n}\right)$ [75], where we introduce

$$
\begin{aligned}
& \gamma_{+}\left(q_{n}\right)=+u_{q_{n}}^{*} w_{+}\left(q_{n}\right)+v_{q_{n}} w_{-}\left(q_{n}\right), \\
& \gamma_{-}\left(q_{n}\right)=-v_{q_{n}}^{*} w_{+}\left(q_{n}\right)+u_{q_{n}} w_{-}\left(q_{n}\right),
\end{aligned}
$$

together with the following constants,

$$
u_{q_{n}}=\sqrt{\frac{\varepsilon\left(q_{n}\right)+\zeta\left(q_{n}\right)}{2 \varepsilon\left(q_{n}\right)}}, \quad v_{q_{n}}=e^{i \phi_{n}} \sqrt{\frac{\varepsilon\left(q_{n}\right)-\zeta\left(q_{n}\right)}{2 \varepsilon\left(q_{n}\right)}},
$$

and $\zeta\left(q_{n}\right)=2 \tilde{t}+(\Delta \varepsilon / 2) \sin q_{n}, e^{i \phi_{n}}=-i \operatorname{sgn}\left[\cos \left(q_{n}\right)\right]$. We note that by using the periodic bulk energies Eq. (8), our approximation is essentially introducing an intensive contribution coming from the added bonds at the ends of the ladder.

Finally, the imbalance Eq. (15) also induces a bulk-edge coupling that can be understood as a hybridization between the edge and bulk orbitals: 


$$
H_{b-e}=\sum_{n, \alpha}\left(g_{n, \alpha}^{l} l^{\dagger}+g_{n, \alpha}^{r} r^{\dagger}\right) \gamma_{\alpha}\left(q_{n}\right)+\text { H.c. }
$$

To obtain the correct bulk-edge couplings $g_{n, \alpha}^{\eta}$, note that the connection between the solutions of the periodic and open chains requires enlarging the number of sites of the periodic chain. To preserve the distance between the edges, one must change the site indexes of operators that form the edge-bulk coupling Eq. (15), and leads to

$$
\begin{aligned}
& g_{n,+}^{l}=-\frac{\Delta \varepsilon}{2 \sqrt{N}} \sin q_{n}\left(u_{q_{n}}-v_{q_{n}}\right), \\
& g_{n,-}^{l}=-\frac{\Delta \varepsilon}{2 \sqrt{N}} \sin q_{n}\left(u_{q_{n}}^{*}+v_{q_{n}}^{*}\right), \\
& g_{n,+}^{r}=\frac{\Delta \varepsilon}{2 \sqrt{N}}(-i)^{(N-1) / 2} \sin \left[\frac{1}{2} q_{n}(N-1)\right]\left(u_{q_{n}}+v_{q_{n}}\right), \\
& g_{n,-}^{r}=\frac{\Delta \varepsilon}{2 \sqrt{N}}(-i)^{(N-1) / 2} \sin \left[\frac{1}{2} q_{n}(N-1)\right]\left(v_{q_{n}}^{*}-u_{q_{n}}^{*}\right) .
\end{aligned}
$$

Interestingly, the Hamiltonian of the Creutz ladder in this formulation, $H_{\text {edge }}+H_{\text {bulk }}+H_{b-e}$, is similar to a noninteracting two-impurity Fano-Anderson model [76], where the impurities correspond to the modes localized at the edges of the Creutz ladder, and the gapless metallic bands are substituted by gapped bulk bands also described by free fermions. This analogy yields an insightful interpretation of the topological quantum phase transition at $\left.(\Delta \varepsilon / \tilde{t})\right|_{c}=4$.

Interpreting the dispersive bulk bands as reservoirs, the hybridization can have two effects: (i) the zero-energy edge modes $\eta=l, r$ may get shifted and broadened, $\varepsilon_{\eta}=0 \rightarrow \Delta \varepsilon_{\eta}-i \Gamma_{\eta} / 2$, and (ii) the bulk fermions mediate an edge-to-edge tunneling. Because of the particle-hole symmetry of the bands, the level shifts cancel, $\Delta \varepsilon_{\eta}=0$, such that the poles representing the edge excitations always correspond to zero energy. On the other hand, the broadening can be expressed as $\Gamma_{\eta}=\sum_{\alpha} J_{\eta, \alpha}\left(\varepsilon_{\eta}\right)$, where we introduce the spectral density for the coupling of the edge states to the bulk bands:

$$
J_{\eta \alpha}(\omega)=2 \pi \sum_{n}\left|g_{n, \alpha}^{\eta}\right|^{2} \delta\left(\omega-\alpha \varepsilon\left(q_{n}\right)\right) .
$$

Accordingly, the broadening of the levels depends on the value of the spectral function at zero energy, $\Gamma_{\eta}=$ $\sum_{\alpha} J_{\eta \alpha}(0)$. For $\Delta \varepsilon<4 \tilde{t}$, the bulk bands Eq. (8) remain gapped, and thus $J_{\eta \alpha}(0)=0$, such that $\Gamma_{l}=\Gamma_{r}=0$, and the edge modes thus remain in-gap bound states. As noted previously, there will be a bulk-mediated tunneling between these in-gap modes:

$$
H_{e-e}=t_{e e} r^{\dagger} l+\text { H.c., }
$$

where we introduce the tunneling strength,

$$
t_{e e}=\sum_{n, \alpha} \frac{g_{n, \alpha}^{r}\left(g_{n, \alpha}^{l}\right)^{*}}{\alpha \varepsilon\left(q_{n}\right)} .
$$

Provided that the bulk bands Eq. (8) remain gapped, this edge-edge tunneling will decrease exponentially with the edge-to-edge distance, such that the topological degeneracy of the ground states Eq. (13) is preserved in the thermodynamic limit. Conversely, when $\Delta \varepsilon=4 \tilde{t}$, the gap vanishes and the exponential localization of the edge states disappears. Moreover, the bulk density of states at zero energy does not vanish anymore, such that $J_{\eta \alpha}(0) \neq 0$, and the edge modes become broadened resonances $\Gamma_{\eta}>0$ instead of the previous bound states.

It is very instructive to understand how the onset of a topological phase transition can be predicted by looking at the effective theory for the edges. This will become very useful in the presence of interactions, where a simple interpretation in terms of observables associated to a topological invariant Eq. (7) for noninteracting systems cannot be applied. Additionally, this result also underlies the importance of preserving all the orbitals in the effective description in terms of $\mathrm{ABC}$, and not simply projecting to the low-energy flat band when trying to describe flat-band effects in fermionic topological insulators.

\section{Bulk-mediated dephasing and edge-edge interactions}

Let us now derive an effective boundary theory in the balanced interacting model $(\Delta \varepsilon=0)$ for intermediate interactions $V_{v} / \tilde{t}$. Therefore, we must analyze the following part, $H_{\pi \mathrm{CH}}=H_{\mathrm{FB}}+V_{\mathrm{nn}}+V_{\mathrm{pt}}+V_{\mathrm{dt}}$, of the Hamiltonian Eq. (39). Although the pair and density-dependent tunnelings in Eqs. (37) and (38) modify the distribution of particlehole pairs in the rungs of the virtual ladder (see Fig. 5), the nearest-neighbor interactions Eq. (36) do not change, since the number of neighboring $\mathrm{ABC}$ that are occupied is preserved under such processes. Therefore, in this limit, the nearest-neighbor interactions Eq. (36) can be substituted by a $c$ number, and only the flat band Eq. (12) and the correlated-tunneling terms Eqs. (37) and (38) have an important effect on the noninteracting ground states Eq. (13). Moreover, as argued below Eq. (38), only the density-dependent tunneling at the edges of the ladder will play a role to determine the order of the translationally invariant ground states.

According to this discussion, we can rearrange these terms as $H_{\pi \mathrm{CH}}=H_{\text {edge }}+H_{\text {bulk }}+H_{b-e}$, where the edge Hamiltonian coincides with Eq. (40) for the noninteracting imbalanced case. In contrast, the bulk Hamiltonian is no longer described by a quadratic fermionic model, but instead by quartic terms that can be understood as some effective spin exchange. This becomes apparent after introducing the $\mathfrak{s} \mathfrak{u}(2)$ algebra, 


$$
\begin{aligned}
& \tilde{T}_{i}^{x}=\frac{1}{2}\left(w_{i,+}^{\dagger} w_{i,-}+w_{i,-}^{\dagger} w_{i,+}\right), \\
& \tilde{T}_{i}^{z}=\frac{1}{2}\left(w_{i,+}^{\dagger} w_{i,+}-w_{i,-}^{\dagger} w_{i,-}\right),
\end{aligned}
$$

which should not be confused with the strong-coupling orbital spin operators of Eq. (31). With this notation, the bulk Hamiltonian becomes

$$
H_{\mathrm{bulk}}=\frac{V_{v}}{4} N+\sum_{i=1}^{N-1} 4 \tilde{t} \tilde{T}_{i}^{z}+\sum_{i=2}^{N-1} 4 \tilde{J} \tilde{T}_{i-1}^{x} \tilde{T}_{i}^{x}
$$

where $\tilde{J}=-V_{v} / 4$, which corresponds to a ferromagnetic Ising model in a transverse field. This model can be solved exactly for periodic boundary conditions [72] by means of a Jordan-Wigner transformation [60], namely,

$\tilde{T}_{i}^{z}=f_{i}^{\dagger} f_{i}-\frac{1}{2}, \quad \tilde{T}_{i}^{x}=\frac{1}{2} f_{i}^{\dagger} e^{i \pi \sum_{j<i} f_{j}^{\dagger} f_{j}}+$ H.c.,

where $f_{i}^{\dagger}=\left(f_{i}\right)^{\dagger}$ are spinless fermionic operators. Considering periodic boundary conditions $f_{N}=f_{1}$, we would obtain the energy bands for single-particle excitations,

$$
\tilde{\varepsilon}_{ \pm}(q)= \pm \tilde{\varepsilon}(q)= \pm 2|\tilde{J}| \sqrt{1+\tilde{\mathfrak{f}}^{2}-2 \tilde{\mathfrak{f}} \cos q},
$$

where we introduce the flatness parameter $\tilde{\tilde{f}}=8 \tilde{t} / V_{v}$, such that we recover perfect flat bands in the noninteracting regime $V_{v}=0$. Here, the quasimomentum is defined within a halved Brillouin zone $q \in(0, \pi]$, such that there is a total of $N$ single-particle modes. Let us remark that, just by looking at this effective theory of the bulk, one would predict a standard quantum phase transition at $|J|=2 \tilde{t}$ onto an orbital ferromagnet that can be described by a local order parameter. However, this theory would predict a featureless disordered phase for $|J|<2 \tilde{t}$, and thus completely miss the topological features of the model. It is thus of paramount importance to include the boundary $\mathrm{ABC}$ in the full description.

To prepare for that, we use a similar approximation to treat the bulk as in the previous section. We note that the above energies have the symmetry $\tilde{\varepsilon}_{\alpha}(+q)=\tilde{\varepsilon}_{\alpha}(-q)$, such that we can move back to the entire Brillouin zone, and construct $\mathrm{ABC}$ momentum operators that respect the open boundary conditions $f_{N}=f_{0}=0$ of a finite ladder by combining the $\pm q$ solutions of the periodic chain. In this case, in order to recover the $N$ single-particle modes, and taking into account that the $q=\pi$ mode yields a trivial sanding wave, we need to enlarge the number of sites in the problem with periodic boundary conditions $N-1 \rightarrow N$, such that the allowed momenta become $q_{n}=(2 \pi / N-1) n \rightarrow q_{n}=(2 \pi / N) n$, with $n \in\left\{1, \ldots, \frac{1}{2} N\right\}$. Accordingly, the mapping

$$
\left(\begin{array}{c}
f\left(+q_{n}\right) \\
f^{\dagger}\left(-q_{n}\right)
\end{array}\right)=\sqrt{\frac{2}{N}} \sum_{j=1}^{N-1} \sin \left(q_{n} j\right)\left(\begin{array}{c}
f_{j} \\
f_{j}^{\dagger}
\end{array}\right)
$$

transforms the bulk Hamiltonian Eq. (52) approximately into

$$
H_{\text {bulk }} \approx \sum_{n=1}^{N / 2} \tilde{\Psi}^{\dagger}\left(q_{n}\right) \tilde{\boldsymbol{B}}\left(q_{n}\right) \cdot \boldsymbol{\sigma} \tilde{\Psi}\left(q_{n}\right)
$$

where $\tilde{\boldsymbol{B}}\left(q_{n}\right)=\left(0,-2 \tilde{J} \sin q_{n}, 4 \tilde{t}+2 \tilde{J} \cos q_{n}\right)$, and $\tilde{\Psi}\left(q_{n}\right)=$ $\left(f\left(q_{n}\right), f^{\dagger}\left(-q_{n}\right)\right)^{t}$ are the Nambu spinors. Diagonalizing this Hamiltonian leads to the bulk curved energy bands Eq. (54), such that $H_{\text {bulk }}=\sum_{n, \alpha} \alpha \tilde{\varepsilon}\left(q_{n}\right) \tilde{\gamma}_{\alpha}^{\dagger}\left(q_{n}\right) \tilde{\gamma}_{\alpha}\left(q_{n}\right)$, where we introduce

$$
\begin{aligned}
& \tilde{\gamma}_{+}\left(q_{n}\right)=+\tilde{u}_{q_{n}}^{*} f\left(q_{n}\right)+\tilde{v}_{q_{n}} f^{\dagger}\left(-q_{n}\right), \\
& \tilde{\gamma}_{-}\left(q_{n}\right)=-\tilde{v}_{q_{n}}^{*} f\left(q_{n}\right)+\tilde{u}_{q_{n}} f^{\dagger}\left(-q_{n}\right),
\end{aligned}
$$

together with the following constants,

$u_{q_{n}}=\sqrt{\frac{\tilde{\varepsilon}\left(q_{n}\right)+\tilde{\zeta}\left(q_{n}\right)}{2 \tilde{\varepsilon}\left(q_{n}\right)}}, \quad v_{q_{n}}=e^{i \tilde{\phi}_{n}} \sqrt{\frac{\tilde{\varepsilon}\left(q_{n}\right)-\tilde{\zeta}\left(q_{n}\right)}{2 \tilde{\varepsilon}\left(q_{n}\right)}}$,

and $\tilde{\zeta}\left(q_{n}\right)=2 \tilde{t}+\tilde{J} \cos q_{n}, e^{i \tilde{\phi}_{n}}=i \operatorname{sgn}\left[\sin \left(q_{n}\right)\right]$. In contrast to the single-particle bulk excitations in the imbalanced case Eq. (44), the fermionic operators Eq. (57) now describe Bogoliubov fermionic excitations [77]. We note again that by using the periodic bulk energies, our approximation is essentially introducing an intensive contribution coming from the added bonds at the ends of the ladder.

Finally, the bulk-edge coupling corresponds to a spindensity interaction:

$$
H_{b-e}=V_{v}\left(n_{l} \tilde{T}_{1}^{x}-n_{r} \tilde{T}_{N-1}^{x}\right) .
$$

We can express this interaction in terms of the Bogoliubov excitations, after using the above Jordan-Wigner transformation, together with Eqs. (55) and (57). In a first approximation, we neglect the Jordan-Wigner string, which allows us to derive a bulk-edge coupling that is analogous to the imbalance-induced hybridization Eq. (46), namely,

$$
H_{b-e}=\sum_{n, \alpha}\left(\tilde{g}_{n, \alpha}^{l} l^{\dagger} l-\tilde{g}_{n, \alpha}^{r} r^{\dagger} r\right) \tilde{\gamma}_{\alpha}\left(q_{n}\right)+\text { H.c. }
$$

Once again, in order to obtain the correct bulk-edge couplings $\tilde{g}_{n, \alpha}^{\eta}$, we have to consider that the connection between the modes of the periodic and open chain require enlarging the number of lattice sites, and thus changing the indexing of the operators in the edge-bulk coupling. This leads to 


$$
\begin{aligned}
& \tilde{g}_{n,+}^{l}=+\frac{V_{v}}{\sqrt{2 N}} \sin q_{n} \tilde{u}_{q_{n}}, \\
& \tilde{g}_{n,-}^{l}=-\frac{V_{v}}{\sqrt{2 N}} \sin q_{n} \tilde{v}_{q_{n}}, \\
& \tilde{g}_{n,+}^{r}=+\frac{V_{v}}{\sqrt{2 N}} \sin \left[q_{n}(N-1)\right] \tilde{u}_{q_{n}}, \\
& \tilde{g}_{n,-}^{r}=-\frac{V_{v}}{\sqrt{2 N}} \sin \left[q_{n}(N-1)\right] \tilde{v}_{q_{n}} .
\end{aligned}
$$

Accordingly, the Creutz-Hubbard ladder in this formulation, $H_{\pi \mathrm{CH}}=H_{\text {edge }}+H_{\text {bulk }}+H_{b-e}$, becomes again similar to a noninteracting two-impurity Fano-Anderson model [76]. In this case, the gapless metallic bands in the FanoAnderson model are substituted by gapped bands of Bogoliubov fermions Eq. (57) due to the lack of conservation of the number of Jordan-Wigner fermions. The bulkedge hybridization Eq. (46) is substituted by a bulk-edge coupling Eq. (60) that does not conserve the number of Jordan-Wigner fermions either, and depends on the population of the edge modes. This analogy allows us to predict the onset of a topological quantum phase transition in the Creutz ladder caused solely by the Hubbard interactions.

Interpreting once more the dispersive bulk bands as reservoirs, the bulk-edge coupling can have two effects: (i) the zero-energy edge modes $\eta=l, r$ may get shifted $\varepsilon_{\eta}=0 \rightarrow \varepsilon_{\eta}=\Delta \varepsilon_{\eta}$ and dephased with a rate $\tilde{\Gamma}_{\eta} / 2$, and (ii) the bulk Bogoliubov excitations can mediate an edgeedge interaction (i.e., density-density interactions between the edge fermions mediated by spin-wave-type excitations). In analogy to the imbalance-induced broadening Eq. (48), the dephasing rate can be expressed as $\tilde{\Gamma}_{\eta}=\sum_{\alpha} \tilde{J}_{\eta \alpha}\left(\varepsilon_{\eta}\right)$, where we introduce the spectral density for the new bulkedge coupling:

$$
\tilde{J}_{\eta \alpha}(\omega)=2 \pi \sum_{n}\left|\tilde{g}_{n, \alpha}\right|^{2} \delta\left(\omega-\alpha \tilde{\varepsilon}\left(q_{n}\right)\right) .
$$

Accordingly, the dephasing of the superposition states depends on the value of this spectral function at zero energy, $\tilde{\Gamma}_{\eta}=\sum_{\alpha} \tilde{J}_{\eta \alpha}(0)$. For $V_{v} / 4<2 \tilde{t}$, the bulk bands Eq. (54) remain gapped, and thus $J_{\eta \alpha}(0)=0$, such that there is no dephasing. In this regime, there are only level shifts and edge-edge interactions described by

$$
H_{e-e}=\Delta \varepsilon_{l} n_{l}+\Delta \varepsilon_{r} n_{r}+U_{e e} n_{l} n_{r}
$$

where we introduce

$\Delta \varepsilon_{\eta}=-\sum_{n, \alpha} \frac{\left|\tilde{g}_{n, \alpha}^{\eta}\right|^{2}}{\varepsilon\left(q_{n}\right)}, \quad U_{e e}=\sum_{n, \alpha} \frac{\tilde{g}_{n, \alpha}^{l} \tilde{g}_{n, \alpha}^{r}}{\varepsilon\left(q_{n}\right)}+$ c.c.

Let us note that, in contrast to the imbalanced case, the particle-hole symmetry does not impose the vanishing of the level shifts. Because of the different bulk-edge coupling, these shifts depend on the filling of the Bogoliubov levels for the bulk ground state $\left|g_{\text {bulk }}\right\rangle=\tilde{\gamma}_{-}^{\dagger}\left(q_{1}\right) \tilde{\gamma}_{-}^{\dagger}\left(q_{2}\right) \ldots$ $\tilde{\gamma}_{-}^{\dagger}\left(q_{N-1}\right)\left|0_{B}\right\rangle$, where $\left|0_{B}\right\rangle$ is the Bogoliubov vacuum, and thus do not vanish.

If we now use a similar argument as for the imbalanceinduced quantum phase transition, we find that (i) the bulk-mediated edge-edge interaction has no effect on the degenerate ground state Eq. (13), since both edge modes are not populated simultaneously for a half-filled system, and (ii) although the zero modes are shifted in energy, the topological degeneracy is preserved, $\Delta \varepsilon_{l}=\Delta \varepsilon_{r}$, and no dephasing occurs provided that the Bogoliubov excitations remain gapped. Conversely, when $|\tilde{J}|=2 \tilde{t}$, or equivalently, $V_{v}=8 \tilde{t}$, dephasing takes place signaling that the edge modes are not well-defined single-particle excitations. This argument thus locates the critical point of the interactioninduced quantum phase transition at $\left.\left(V_{v} / \tilde{t}\right)\right|_{c}=8$, which is represented by a red circle in the phase diagram of Fig. 1.

\section{E. Phase diagram of the Creutz-Hubbard ladder}

Our considerations in the above sections has already allowed us to determine the possible phases of the model and their phase boundaries in certain parameter regimes. In the following, we lay out the full phase diagram of the model in the $\left[(\Delta \varepsilon / \tilde{t}),\left(V_{v} / \tilde{t}\right)\right]$ plane using DMRG calculations.

Our DMRG code is based on matrix product states and uses a two-site optimization scheme. It is built on the Abelian Symmetric Tensor Networks Library (developed in collaboration with the group of Montangero in Ulm) and capable of implementing multiple Abelian symmetries, such as particle-number conservation. We consider lattices up to $N=256$ sites and virtual bond dimensions of up to $m=200$.

The analytic and numerical results for the phase diagram, collected in Fig. 1, are described in the following sections.

\section{Topological insulator to orbital paramagnet phase transition}

As shown in Sec. III B, the mapping of the Creutz ladder onto a quantum Ising ladder allows us to predict a critical line Eq. (29) separating the topological insulator (TI) and the orbital paramagnet (OPM) for sufficiently weak interactions. This critical line is represented by a red dashed line that starts to form the point $\Delta \varepsilon=4 \tilde{t}, V_{v}=0$ in Fig. 1 .

The Ising transverse magnetization, or, equivalently, the density imbalance between the legs of the Creutz ladder Eq. (28), can serve as a good indicator for this quantum phase transition that can be easily calculated numerically using the DMRG code. We can determine the critical line by studying the divergence of the imbalance susceptibility, $\chi_{\Delta n}=\partial\langle\Delta n\rangle / \partial(\Delta \varepsilon / \tilde{t})$ [Fig. 6 (top)].

As an alternative means of identifying the TI phase, we can study the behavior of the ground-state degeneracy in 

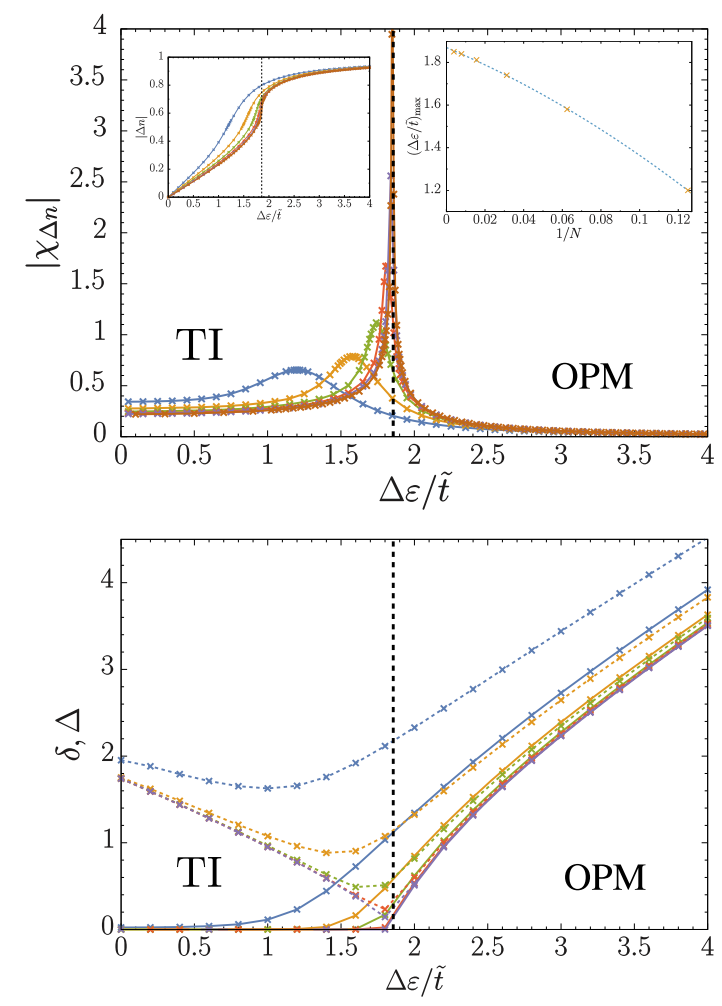

FIG. 6. Paramagnetic susceptibility and energy gaps along the TI-OPM transition. Top: The divergence of the magnetization susceptibility $\chi_{\Delta n}$ with growing system size indicates the critical point for a cut through the phase diagram at $V_{v}=4.0$ (left-hand inset: occupation imbalance $\Delta n$; right-hand inset: fitted finite-size scaling of the susceptibility maxima assuming up to second-order corrections, $\left.\Delta \varepsilon_{c}(N)=\Delta \varepsilon_{c}\left(1+a N^{-1}+b N^{-2}\right)\right)$. Bottom: The dashed lines show the finite-size results for the energy gap $\Delta$, which is nonzero in both the TI and the OPM phase. The quantity $\delta$ (solid lines), on the contrary, is zero in the TI phase due to the presence of zero-energy modes, but achieves a nonvanishing value in the OPM phase. Blue, $N=8$; orange, $N=16$; green, $N=32$; red, $N=64$; violet, $N=128$; brown, $N=256$. The vertical line (black) indicates the transition point $\left(\Delta \varepsilon_{c}=1.857\right)$.

the Creutz-Hubbard model with variable filling. We therefore introduce the single- and two-particle energy gaps

$$
\begin{array}{r}
\Delta=\lim _{N \rightarrow \infty} \frac{1}{2}[E(N+2)+E(N-2)-2 E(N)], \\
\delta=\lim _{N \rightarrow \infty}[E(N+1)+E(N-1)-2 E(N)],
\end{array}
$$

where $E(x)$ is the ground-state energy of a system with $x$ particles. It can be shown that the two quantities coincide for gapless systems $(\Delta=\delta=0)$ and conventional insulators $(\Delta=\delta \neq 0)$. In a topological insulator, however, $\delta=0$ due to the presence of zero-energy edge modes while $\Delta \neq 0$ measures (half) the band gap. In Fig. 6 (bottom), we show that the predicted behavior is indeed observed.
The critical points obtained through these different observables are represented by yellow stars in the lefthand part of Fig. 1. As can be seen from these results, the analytical prediction of the phase boundary Eq. (29) is reasonably accurate even for quite large interactions, where the exact critical line given by DMRG departs from a straight line, and bends up.

\section{Orbital ferromagnet to orbital paramagnet phase transition}

In Sec. IIIC, we introduce an effective orbital Ising model in the limit of very strong interactions, which allows us to predict a critical line Eq. (33) separating the orbital ferromagnet (OFM) and orbital paramagnet (OPM). This critical line is represented by a yellow dashed line in Fig. 1 .

Indeed, by measuring the paramagnetic and ferromagnetic magnetization $\left[\left\langle T_{i}^{z}\right\rangle\right.$ and $\left\langle T_{i}^{y}\right\rangle$ in Eq. (31)], we confirm that these quantities scale equally, and identify the phasetransition point also for finite interactions (Fig. 7). Technically, we determine the paramagnetic magnetization by measuring the fermionic observable that defines $T_{i}^{z}$, which is proportional to the leg density imbalance discussed above [see Eq. (31)]. In order to avoid problems due to incomplete symmetry breaking when studying the ferromagnetic order parameter $\left\langle T_{i}^{y}\right\rangle$ (i.e., between the possible alignments in the ferromagnetic phase), we determine instead the zero-momentum component of the orbital magnetic structure factor,

$$
S_{T_{y} T_{y}}(k)=\frac{1}{N^{2}} \sum_{i, j} e^{i k(i-j)}\left\langle T_{i}^{y} T_{j}^{y}\right\rangle,
$$

which yields the desired ferromagnetic magnetization in the thermodynamic limit, $\left\langle T_{i}^{y}\right\rangle=\left[S_{T_{y} T_{y}}(0)\right]^{1 / 2}$.

We observe for both quantities an Ising-like scaling, which differs from the strong-coupling prediction only by a renormalization of the critical point and of the maximum ferromagnetic magnetization (cf. Fig. 7).

The critical points obtained through these magnetizations are represented by yellow stars in the right-hand part of Fig. 1. As can be seen from these results, the analytical prediction of the phase boundary Eq. (29) is reasonably accurate even for moderate interactions.

\section{Topological insulator to orbital ferromagnet phase transition}

In Sec. III D, we derive an interesting connection between the balanced Creutz-Hubbard ladder for intermediate interactions and a Fano-Anderson-type model, which allows us to predict the extension of the topological phase along the $\left[0,\left(V_{v} / \tilde{t}\right)\right]$ axis of the phase diagram until a critical point $\left(V_{v} / \tilde{t}\right)=8$. Beyond this point, the long-range ordered orbital Ising magnet sets in, and the topological 

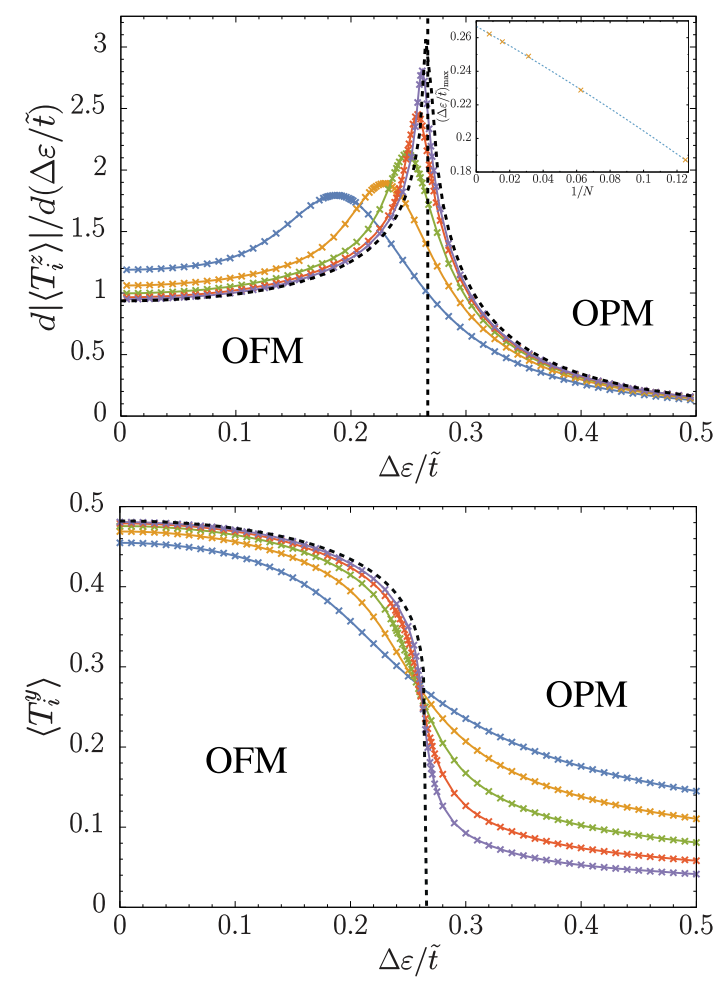

FIG. 7. Ferromagnetic and paramagnetic magnetization along the OFM-OPM transition. Top: Paramagnetic magnetization susceptibility for a cut through the phase diagram at $V_{v}=16 \tilde{t}$ and different system sizes [inset: fitted finite-size scaling of the susceptibility maxima assuming up to second-order corrections, $\left.\Delta \varepsilon_{c}(N)=\Delta \varepsilon_{c}\left(1+a N^{-1}+b N^{-2}\right)\right]$. Bottom: Ferromagnetic magnetization along the same line. Blue, $N=8$; orange, $N=16$; green, $N=32$; red, $N=64$; violet, $N=128$. The light blue vertical line (dashed) in the top figure indicates the critical point (here, $\Delta \varepsilon_{c} / \tilde{t}=0.266$ ). The black dashed curves indicate the analytical predictions of an Ising model with the same critical point (and a saturation of the ferromagnetic magnetization $\left.\left\langle T_{i}^{y}\right\rangle_{\max }=0.48\right)$. The effective Ising model in Eq. (31) suggests $\Delta \varepsilon_{c} / \tilde{t}=0.25$ and $\left\langle T_{i}^{y}\right\rangle_{\max }=0.5$.

edge modes disappear into the bulk. This critical point is represented by a red circle in Fig. 1.

The numerical analysis (Fig. 8) confirms the validity of the effective Ising model derived in Eq. (52), and the exact location of this critical point. Moreover, in the case of finite imbalance $(\Delta \varepsilon \neq 0)$, the divergence of the paramagnetic susceptibility serves as a criterion for the determination of the phase boundary (see inset Fig. 8). The critical points obtained by these means are represented by yellow stars in the middle part of Fig. 1.

\section{Conformal field theories for the critical lines and entanglement spectrum for the phases}

Thus far, we have used a conventional condensed-matter approach to explore numerically the phase diagram of the model, which is based on exploiting energy gaps, susceptibilities, and correlation functions to identify phases with

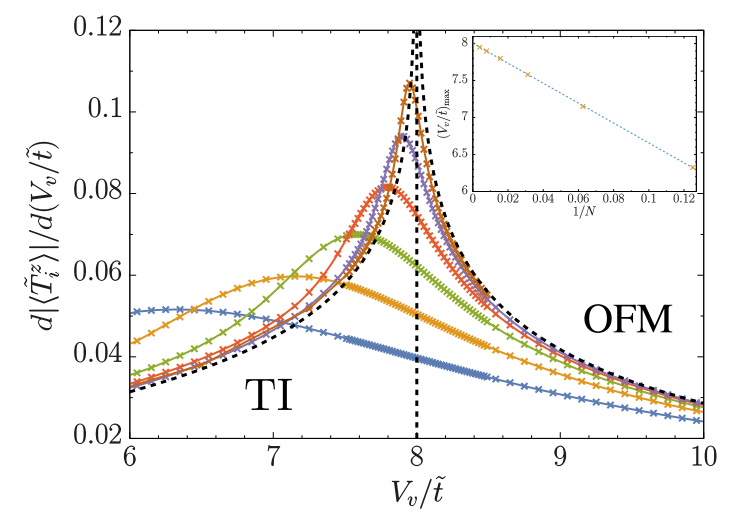

FIG. 8. Paramagnetic magnetization susceptibility along the TI-OFM transition. Blue, $N=8$; orange, $N=16$; green, $N=32$; red, $N=64$; violet, $N=128$; brown, $N=256$. The black dashed lines indicate the TD result in a TFIM model. Inset: The finite-size scaling of the maxima of the susceptibility yields $\left(V_{v} / \tilde{t}\right)_{c \text {,num }}=8.003$ [here, fitted via $V_{v, c \text {,num }}(N)=$ $\left.V_{v, c, \text { num }}\left(1+a N^{-1}\right)\right]$, in good agreement with the analytical result $\left(V_{v} / \tilde{t}\right)_{c}=8$.

long-range order or symmetry-protected topological phases, and critical lines that separate them. An alternative approach, based on the ground-state entanglement, has recently become a complementary method to understand the phase diagram of quantum many-body models [78]. For instance, the pairwise concurrence "susceptibility" can serve as a probe to localize quantum critical points [79], displaying a scaling behavior similar to that of certain observables in the more conventional condensed-matter approach. Other entanglement measures can also serve as probes of quantum criticality, as occurs for the block entanglement entropy $S(l)=-\operatorname{Tr}\left\{\rho_{l} \log \rho_{l}\right\}$, where $\rho_{l}=$ $\operatorname{Tr}_{L-l}\left\{\left|\varepsilon_{\mathrm{gs}}\right\rangle\left\langle\varepsilon_{\mathrm{gs}}\right|\right\}$ is the reduced density matrix of the left block with $l$ sites for a bipartition of a chain of $L$ sites. Remarkably enough, not only does the block entanglement entropy serve as a probe of criticality due to its divergence at a phase transition, but its scaling with the system size also reveals the central charge $c$ of the conformal field theory underlying the critical behavior [80,81]. For a critical system with open boundary conditions, the block entanglement entropy scales as

$$
S(l)=\frac{c}{6} \ln \left(\frac{2 L}{\pi} \sin \frac{\pi l}{L}\right)+a,
$$

where we introduce a nonuniversal constant $a$. Since such entanglement entropy can be easily recovered from our MPS numerical results, calculating the central charge of the different critical lines of our phase diagram can serve as an additional confirmation of our previous derivations.

In Sec. III B, we argue that the synthetic Creutz ladder for sufficiently weak interactions can be understood as a couple of Ising models of length $L=N$ with a 
renormalized transverse field. Accordingly, the corresponding CFT should have central charge of $c=1 / 2+$ $1 / 2=1$, such that we would expect the scaling $S(l)=$ $\frac{1}{6} \ln [(2 N / \pi) \sin (\pi l / N)]+a$. This is a natural connection to the noninteracting regime of Sec. III, where we argue that the phase transition can be understood in terms of a Dirac fermion with a Wilson mass in lattice gauge theories. This Wilson fermion becomes massless at the critical point, and corresponds to the CFT of a single massless Dirac fermion with central charge $c=1$.

For the strongly interacting regime of Sec. III C, we show that the OFM-OPM quantum phase transition can be predicted in terms of a single Ising model of length $L=N$ in a transverse field. Accordingly, the corresponding CFT should have central charge of $c=1 / 2$, and $S(l)=$ $\frac{1}{12} \ln [(2 N / \pi) \sin (\pi l / N)]+\tilde{a}$. In contrast to the previous case, the critical phenomenon is governed by the CFT of a single Majorana fermion with central charge $c=1 / 2$.

Finally, in the intermediate interacting regime of Sec. III D, we argue that the relevant physics to understand the TI-OFM phase transition is by approximating a complicated nonstandard Hubbard model with a simpler quantum impurity model for the edges coupled to a bulk of spins described by yet another Ising model of $L=N-1$ sites in a transverse field. Technically, however, we determine the entanglement entropy between the $N$ physical sites in the original basis and therefore measure $S(l)=\frac{1}{12} \ln [(2 N / \pi) \sin (\pi l / N)]+a^{\prime}$ for a system of length $L=N$, corresponding to the CFT of a single Majorana fermion with a central charge of $c=1 / 2$.

We confirm the above predictions through the numerical determination of the central charge along the critical lines in three representative cases (see Fig. 9). We find central charge values agreeing with $c=1 / 2$ for the TI-OFM and the OFM-OPM transition. The charge $c=1$ along the TI-OPM transition originates from the hybrid nature of the Ising model describing it [see Eq. (20)]. Building on these results, we depict the central charges of the three critical lines of our phase diagram in Fig. 1. Interestingly, the massless Dirac fermion $c=1$ governing the topological phase transition TI-OPM for sufficiently weak interactions is split into a pair of massless Majorana fermions $c=\frac{1}{2}+\frac{1}{2}$ at the tricritical point, each of which governs the critical properties of the TI-OFM and OFM-OPM quantum phase transitions, respectively. At this tricritical point, the central charge is conserved, and the two Majorana fermions paired to yield the Dirac fermion governing the TI-OPM phase transition become unpaired and describe individually the critical properties of the two other phase transitions of the model.

In the previous sections, we provide several indicators of the nontrivial topological nature of the TI phase, such as the resilience of the zero-energy edge modes as interactions are switched on (see Sec. III D), or the different single- and twoparticle gaps [Eq. (66)] that distinguish topological and nontopological phases (see Sec. III E). Let us now provide

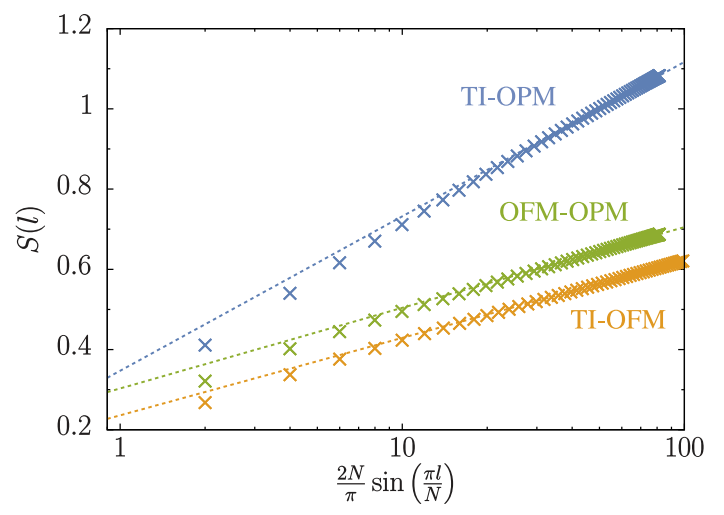

FIG. 9. Scaling of the entanglement entropy for critical points on the different transition lines. The prefactor $c$ in $S(l)=$ $(c / 6) \ln [(2 N / \pi) \sin (\pi l / N)]+$ const identifies the central charge of a critical phase. Here, we show the entanglement entropy in systems with $N$ sites and fit the data for $N / 4<l \leq N / 2$. The fitting results for the TI-OPM transition yield $c=1.003$ for $V_{v} / \tilde{t}=4.0, \Delta \varepsilon / \tilde{t}=1.857, N=128$ (blue line), the OFM-OPM transition yield $c=0.524$ for $V_{v} / \tilde{t}=16, \Delta \varepsilon=0.266, N=128$ (green line), and the TI-OFM transition yield $c=0.503$ for $V_{v} / \tilde{t}=0, \Delta \varepsilon / \tilde{t}=8, N=256$ (yellow line). All these numerical fits agree considerably well with the model predictions $c \in\{1,1 / 2,1 / 2\}$.

further evidence by using entanglement properties of the ground state. In particular, a strong signature of the presence of topological order can be extracted from the study of the entanglement spectrum (ES) [82]. Similarly to the case of the entanglement entropy, to define the ES we take a

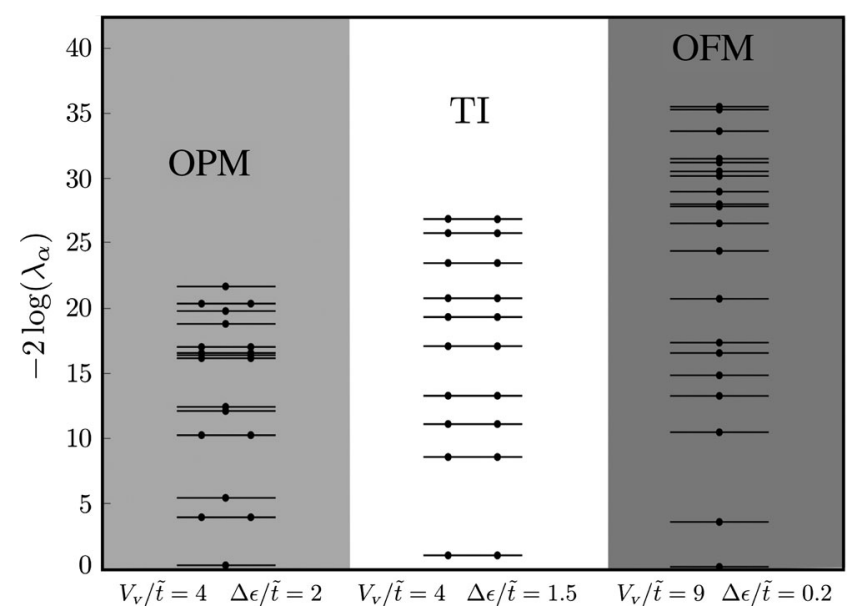

FIG. 10. Degeneracies of the entanglement spectrum for different phases. For a ladder of length $L=128$ and for a bipartition in the half chain, the 20 lower eigenvalues of the ES are depicted for the three different phases. The dots represent the degeneracy of the corresponding eigenvalue. In the TI phase, for $V_{v} / \tilde{t}=4$ and $\Delta_{\varepsilon} / \tilde{t}=1.5$ the eigenvalues are all doubly degenerate. In the OPM phase, for $V_{v} / \tilde{t}=4$ and $\Delta_{\varepsilon} / \tilde{t}=2$, and in the OFM phase for $V_{v} / \tilde{t}=9$ and $\Delta_{\varepsilon} / \tilde{t}=0.2$, almost all the eigenvalues are not degenerate. We find the same behavior elsewhere in phase space. 
(a)

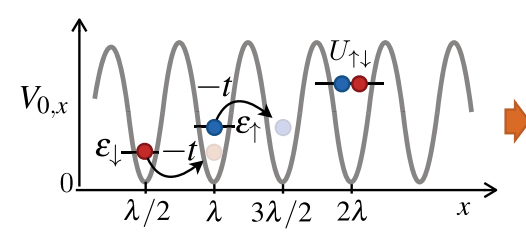

(b)

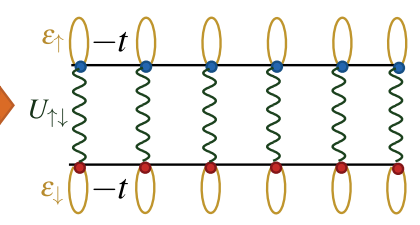

(c)

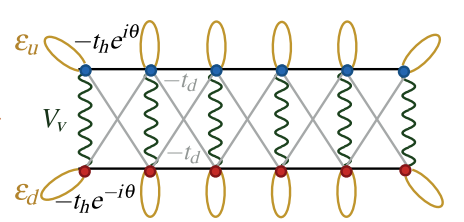

FIG. 11. Ladder scheme for a binary hyperfine mixture of ultracold atoms in an optical lattice. (a) Atoms in two hyperfine states, $|\uparrow\rangle=\left|F=I-\frac{1}{2}, M\right\rangle$ (blue circles) and $|\downarrow\rangle=\left|F^{\prime}=I+\frac{1}{2}, M^{\prime}\right\rangle$ (red circles), are trapped at the minima of an optical lattice. At low temperatures, the kinetic energy of the atoms can be described as tunneling of strength $-t$ between the lowest energy levels $\varepsilon_{\uparrow}, \varepsilon_{\downarrow}$ of neighboring potential wells. Additionally, the $s$-wave scattering of the atoms leads to contact interactions of strength $U_{\uparrow \downarrow}$ whenever two fermionic atoms of different internal state meet on the same potential well. (b) Ladder representation of the binary mixture, where each hyperfine state corresponds to a different leg of the ladder. The tunneling (on-site energy) is represented by solid lines linking neighboring (same) sites within each leg, whereas the on-site interactions are represented by wavy lines among two neighboring sites that belong to different legs. (c) Creutz-Hubbard model obtained by including a Peierls phase in the intraleg tunnelings $-t \rightarrow \pm-t_{h} e^{ \pm i \theta}$, and introducing interleg tunnelings $-t_{d}$ represented by solid lines along diagonal rungs of the ladder.

bipartition of the ladder and the resulting Schmidt decomposition of the ground state $|\psi\rangle=\sum_{i} \lambda_{i}\left|\psi_{L}^{i}\right\rangle \otimes\left|\psi_{L-l}^{i}\right\rangle$, where $\left|\psi_{L}^{i}\right\rangle$ and $\left|\psi_{L-l}^{i}\right\rangle$ are basis vectors of the two parts, satisfying the orthogonality condition $\left\langle\psi_{l}^{j} \mid \psi_{L-l}^{i}\right\rangle=\delta_{i j}$, whereas the $\lambda_{i}$ are the Schmidt values. By convention, the ES is defined as a logarithmic rescaling of the Schmidt values, $-2 \log \left(\lambda_{i}\right)$, and it can be again extracted straightforwardly from MPS calculations. As originally pointed out in Ref. [83] in the context of the characterization of the Haldane phase of Heisenberg-type magnets, the degeneracy of the ES robustly identifies the symmetries protecting the topological phase. As shown in Fig. 10 in the imbalanced Creutz-Hubbard model, the ES in the TI phase clearly shows doubly degenerate eigenvalues, whereas in the OPM and OFM phases the ES is trivial and almost completely nondegenerate. This supports the topological nature of the wide region of the phase diagram labeled as TI (see Fig. 1), and demonstrates that the topological insulating phase survives to considerably strong interactions.

\section{SYNTHETIC CREUTZ-HUBBARD LADDER}

In this section, we discuss an experimental setup capable of implementing the imbalanced Creutz-Hubbard Hamiltonian Eq. (3). We focus on a particular set of microscopic couplings that can be realized with ultracold atoms in optical lattices by exploiting the tools of laser-assisted tunneling.

\section{A. Cold-atom Creutz-Hubbard ladder}

We consider a cubic state-independent optical lattice that traps a two-component atomic gas with hyperfine states $|\uparrow\rangle=$ $\left|F=I-\frac{1}{2}, M\right\rangle,|\downarrow\rangle=\left|F^{\prime}=I+\frac{1}{2}, M^{\prime}\right\rangle$ [see Fig. 11(a)]. In the Wannier basis, the Hamiltonian corresponds to the standard Hubbard model [25], namely,

$$
\begin{aligned}
H= & \sum_{i=1}^{N} \sum_{\sigma=\uparrow, \downarrow}\left(-t f_{i+1, \sigma}^{\dagger} f_{i, \sigma}+\frac{\varepsilon_{\sigma}}{2} n_{i, \sigma}+\text { H.c. }\right) \\
& +\sum_{i} U_{\uparrow \downarrow} n_{i, \uparrow} n_{i, \downarrow},
\end{aligned}
$$

where the fermionic operators $f_{i, \sigma}^{\dagger}, f_{i, \sigma}$ create and annihilate an atom in the electronic state $\sigma$ at site $i$ of the lattice, and $n_{i, \sigma}=f_{i, \sigma}^{\dagger} f_{i, \sigma}$ are the number operators. In this equation, $t$ is the tunneling strength of atoms between neighboring potential wells along the $x$ axis, $\varepsilon_{\sigma}$ stand for the energies of the electronic levels, and $U_{\uparrow \downarrow}$ corresponds to the on-site interaction strength due to $s$-wave scattering. As customary [22], tunnelings and interactions of a longer range, as well as tunnelings along the $y$ and $z$ axes, are neglected in this expression. This is justified for sufficiently deep optical lattices $V_{0, y}, V_{0, z} \gg V_{0, x} \gg E_{R}$, where $V_{0 \alpha}$ are the corresponding amplitudes of the optical potential $V(\mathbf{r})=$ $\sum_{\alpha=x, y, z} V_{0 \alpha} \sin ^{2}\left(k r_{\alpha}\right), E_{R}=k^{2} / 2 m$ is the recoil energy, and $k$ is the wave vector of the retroreflected laser beams forming the optical lattice. In this regime, the parameters of Eq. (69) can be expressed as

$$
\begin{aligned}
t & =\frac{4 E_{R}}{\sqrt{\pi}}\left(\frac{V_{0, x}}{E_{R}}\right)^{3 / 4} e^{-2 \sqrt{V_{0 x} / E_{R}}}, \\
U_{\uparrow \downarrow} & =\sqrt{\frac{8}{\pi}} k a_{\uparrow \downarrow} E_{R}\left(\frac{V_{0 x} V_{0 y} V_{0 z}}{E_{R}}\right)^{1 / 4},
\end{aligned}
$$

where $a_{\uparrow \downarrow}$ is the $s$-wave scattering length for the collision of two atoms in the two different hyperfine states.

The first step towards a possible implementation of the Creutz-Hubbard model based on Eq. (69) is to represent the legs of the ladder by the two hyperfine states $[84,85]$ [see Fig. 11(b)]. This might be interpreted as the smallest 
possible synthetic dimension along which real [86] or complex [87] tunnelings can be implemented via Raman transitions, leading to recent experiments realizing synthetic gauge fields $[54,55]$. Unfortunately, the Creutz ladder involves more-complicated tunnelings [see Fig. 11(c)] that cannot be directly obtained using this scheme. One possibility to implement the required tunnelings could be to combine bichromatic optical lattices with additional Raman transitions and a staggered optical potential, which allows for a very flexible toolbox to realize spin-dependent tunnelings [85]. However, in view of the success of experimental schemes based on periodic modulation of the lattice $[32,33]$ that can be understood in terms of Floquet engineering [88], we hereby present an alternative scheme to achieve the desired Hamiltonian combining periodic modulations with a generalization of the Raman-assisted scheme [27] applied to a spin-independent optical lattice.

\section{State-independent energy gradient}

We consider a linear energy gradient that tilts the optical lattice independently of the hyperfine state. Such a linear gradient can be obtained by accelerating the lattice, or by exploiting the ac-Stark effect, and yields

$$
H_{\mathrm{tilt}}=\sum_{i, \sigma} \Delta i f_{i, \sigma}^{\dagger} f_{i, \sigma} .
$$

Note that we have considered the regime $\Delta \ll V_{0 x}$, such that the gradient does not modify the intersite terms of the original Hubbard model Eq. (69), but only leads to the local on-site term Eq. (71) in the Wannier basis. Moreover, we impose $t \ll \Delta$, such that the tilt inhibits the original intraleg tunneling in Fig. 11(b). The goal now is to reactivate the tunneling against this gradient, by generalizing the ideas of schemes based on Raman transitions [27], or on periodic modulations [89] with additional shallower optical lattices [32].

\section{Raman-assisted tunneling}

We consider three additional laser beams. These lead to a couple of Raman two-photon excitations that assist the interleg tunnelings [see Fig. 12(a)]. A first pair of laser beams with frequencies $\omega_{1}, \omega_{2}$ assists the interleg tunnelings in Fig. 12(b) by virtually populating an excited state. The effect of the lasers is twofold: they provide the energy to overcome the potential offset in the tunneling process $\omega_{1}-\omega_{2}=$ $\left(\varepsilon_{\downarrow}-\varepsilon_{\uparrow}\right)+\Delta$, and they also induce a recoil kick $\delta k=$ $\left(\mathbf{k}_{1}-\mathbf{k}_{2}\right) \cdot \mathbf{e}_{x}$ along the $x$ axis, thus allowing the tunneling to occur (i.e., overlap of neighboring Wannier functions). The other pair of laser beams assists the interleg tunneling in Fig. 12(c) provided that $\omega_{1}-\omega_{3}=\left(\varepsilon_{\uparrow}-\varepsilon_{\downarrow}\right)+\Delta$, also yielding a recoil kick $\delta \tilde{k}=\left(\mathbf{k}_{1}-\mathbf{k}_{3}\right) \cdot \mathbf{e}_{x}$. Altogether, these Raman-assisted terms lead to

$$
H_{\text {Raman }}=\sum_{i} \frac{1}{2}\left(\Omega f_{i+1, \downarrow}^{\dagger} f_{i, \uparrow}+\tilde{\Omega} f_{i+1, \uparrow}^{\dagger} f_{i, \downarrow}\right)+\text { H.c. },
$$

where we introduce the Raman-assisted tunneling strengths

$$
\Omega=\Omega_{12} e^{-(\pi / 4) \sqrt{V_{0 x} / E_{R}}}, \quad \tilde{\Omega}=\Omega_{13} e^{-(\pi / 4) \sqrt{V_{0 x} / E_{R}}},
$$

which are expressed in terms of the respective two-photon Rabi frequencies $\Omega_{12}, \Omega_{13}$ corresponding to the particular Raman transition, and an exponential term due to the laserassisted overlap of the neighboring Wannier orbitals, which has been calculated using a Gaussian approximation. We consider the limit where the Raman-assisted tunneling strengths are set to be equal $\Omega=\tilde{\Omega}$. To arrive at these expressions, we consider again the regime of deep optical lattices, and that $\delta k \cdot \lambda=2 \pi n, \delta \tilde{k} \cdot \lambda=2 \pi \tilde{n}$, where $n, \tilde{n} \in \mathbb{Z}$ (a)

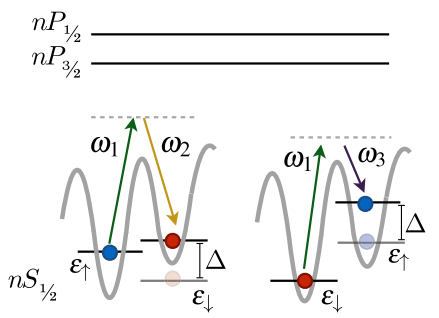

(b)

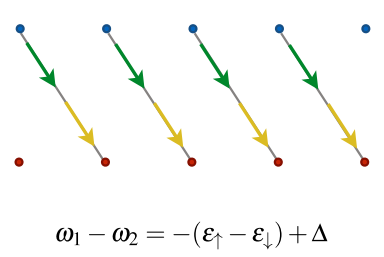

(c)

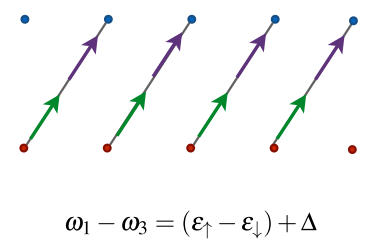

FIG. 12. Raman-assisted tunneling scheme for a binary hyperfine mixture of ultracold atoms in an optical lattice. (a) Atoms in a given hyperfine state from the ground-state manifold $n S_{1 / 2}$ can tunnel to the neighboring potential well changing its hyperfine state by means of a Raman transition that virtually populates an excited state from the manifolds $n P_{1 / 2}, n P_{3 / 2}$. (b) The interleg tunneling of $|\uparrow\rangle$ (blue circles) to $|\downarrow\rangle$ (red circles) is mediated by a pair of lasers providing the energy necessary to overcome the offset $\omega_{1}-\omega_{2}=\left(\varepsilon_{\downarrow}-\varepsilon_{\uparrow}\right)+\Delta$, and a recoil kick to assist the tunneling. (c) The interleg tunneling of $|\downarrow\rangle$ (red circles) to $|\uparrow\rangle$ (blue circles) is mediated by a pair of lasers providing the energy necessary to overcome the offset $\omega_{1}-\omega_{3}=\left(\varepsilon_{\uparrow}-\varepsilon_{\downarrow}\right)+\Delta$, and a recoil kick to assist the tunneling. 
(a)
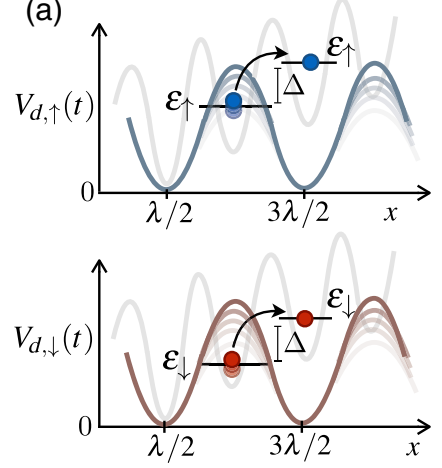

(b)

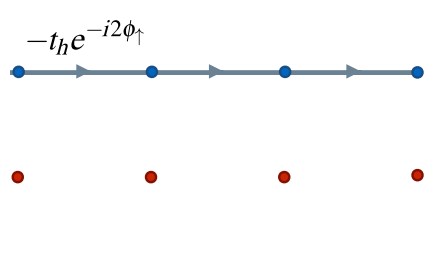

$\omega_{d, \uparrow}=\Delta / 2$ (c)

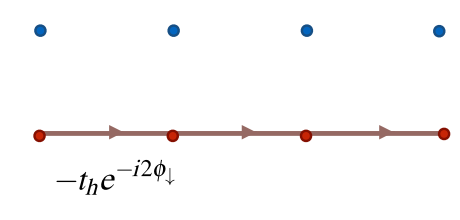

FIG. 13. Photon-assisted tunneling scheme for a binary hyperfine mixture of ultracold atoms in an optical lattice. (a) Atoms in a given hyperfine state can tunnel to the neighboring potential well preserving its hyperfine state by absorbing energy from an additional optical lattice whose intensity is periodically modulated. (b) The intraleg tunneling of $|\uparrow\rangle$ (blue circles) is activated by the weak modulated lattice with $\omega_{d, \uparrow}=\Delta / 2$, such that the tunneling picks the modulation phase $e^{-i 2 \phi_{\uparrow}}$. (c) The intraleg tunneling of $|\downarrow\rangle$ (red circles) is activated by the weak modulated lattice with $\omega_{d, \downarrow}=\Delta / 2$, such that the tunneling picks the modulation phase $e^{-i 2 \phi_{\downarrow}}$.

and $\lambda$ is the wavelength of the retroreflected laser beam that leads to the original optical lattice. This can be achieved by controlling the angle of the Raman lasers with respect to the $x$ axis. More importantly, we assume that the two-photon Rabi frequencies fulfil $\left|\Omega_{12}\right|,\left|\Omega_{13}\right| \ll \Delta$, such that on-site Raman transitions are highly off resonant, and can be neglected.

Let us note that, by avoiding a state-dependent optical lattice that traps each of the two components differently, our scheme is not subjected to the heating problems that become especially troublesome for fermionic atoms [90].

\section{Intensity-modulated optical lattice}

To obtain the complex horizontal hopping in Fig. 11(c), we must reactivate the intraleg tunneling inhibited by the lattice tilting [Eq. (71)] and dress it with the correct Peierls phases. This can be achieved by introducing a periodic driving provided by an additional optical lattice with a modulated intensity [see Fig. 13(a)], namely,

$$
H_{\text {driving }}=\sum_{i, \sigma} V_{d, \sigma}(t) \sin ^{2}\left(k_{d} x_{i}^{0}\right) f_{i, \sigma}^{\dagger} f_{i, \sigma},
$$

where the intensity of the ac-Stark shifts for each of the hyperfine states is modulated periodically in time, $V_{d, \sigma}(t)=V_{d, 0} \sin \left(\omega_{d, \sigma} t-\phi_{\sigma}\right)$, with frequency $\omega_{d, \sigma}$ and a phase $\phi_{\sigma}$. In analogy with the lattice tilting Eq. (71), we assume that the amplitude fulfills $V_{d, 0} \ll V_{0 x}$, such that we can neglect any periodic modulation on the bare tunneling of the original Hubbard model Eq. (70), and consider instead a periodic driving of the on-site energies Eq. (74). More importantly, since this lattice must be very shallow, one can minimize the scattering of photons from the excited state in the calculation of the two-photon ac-Stark shift, which can lead to the aforementioned problematic heating effects in the opposite regime of deep state-dependent optical lattices [90]. As an interesting alternative, we note that a time-dependent magnetic-field gradient [91] can also be exploited to obtain state-dependent time-periodic modulations.

\section{Effective Creutz-Hubbard Hamiltonian}

Now that the ingredients have been introduced, let us show how the synthetic Creutz-Hubbard model is obtained. If the wavelength of this intensity-modulated lattice is twice that of the original lattice, $\lambda_{d}=2 \lambda$, only the even sites will be subjected to the periodic modulation [see Fig. 13(a)]. Moreover, if the resonance condition is met, namely, $n \omega_{d, \sigma}=\Delta$ for $n \in \mathbb{Z}$, the nearest-neighbor tunneling can be restored by absorbing energy quanta from the periodic drive. By setting $\omega_{d, \sigma}=\Delta / 2$, the intraleg tunnelings acquire the phase of the corresponding drivings [see Figs. 13(b) and 13(c)], and lead to

$$
H_{h}=-t \mathfrak{J}_{2}\left(\frac{V_{d, 0}}{\Delta}\right) \sum_{i, \sigma} e^{-i 2 \phi_{\sigma}} f_{i+1, \sigma}^{\dagger} f_{i, \sigma}+\text { H.c., }
$$

where $\mathfrak{J}_{2}(x)$ is the second-order Bessel function of the first class. Let us note that the phases of the periodic modulations must fulfil $\phi_{\uparrow}=-\phi_{\downarrow}$, and that the Raman-assisted tunneling Eq. (72) will get off-resonantly modified by this driving, leading to the final interleg tunneling,

$H_{d}=\frac{\Omega}{2} \mathfrak{J}_{0}\left(\frac{V_{d, 0}}{\Delta}\right) \sum_{i}\left(f_{i+1, \downarrow}^{\dagger} f_{i, \uparrow}+f_{i+1, \uparrow}^{\dagger} f_{i, \downarrow}\right)+$ H.c.,

where $\mathfrak{J}_{0}(x)$ is the zero-order Bessel function. We finally note that the on-site Raman transitions, which were previously neglected under a rotating-wave approximation $\left|\Omega_{12}\right|,\left|\Omega_{13}\right| \ll \Delta$, can also become activated due to the intensity-modulated lattice. This contributes with a spurious vertical interleg tunneling: 


$$
\begin{aligned}
H_{v}= & \frac{\Omega_{12}}{2} \mathfrak{\Im}_{2}\left(\frac{2 V_{d, 0}}{\Delta} \sin \left[\frac{1}{2}\left(\phi_{\uparrow}-\phi_{\uparrow}\right)\right]\right) \sum_{i} f_{2 i, \downarrow}^{\dagger} f_{2 i, \uparrow} \\
& + \text { H.c. }
\end{aligned}
$$

At this point we should stress that the above derivation does not pose any constraint on the ratio $V_{d, 0} / \Delta$. Hence, we can set this ratio such that we hit a zero of the corresponding Bessel function, e.g., $2 V_{d, 0} \sin \left(\phi_{\uparrow}-\phi_{\uparrow} / 2\right)=5.1356 \Delta$, which yields a coherent destruction of the spurious tunneling against the energy offset [92], such that we can make $H_{v} \approx 0$.

All the ingredients discussed so far combine to give us the desired laser-assisted tunneling that implements the Creutz-ladder kinetic Hamiltonian $H_{C}=H_{h}+H_{d}$ [Eq. (1)]. This becomes clear after the mapping

$$
f_{i, \uparrow} \rightarrow c_{i, u}, \quad f_{i, \downarrow} \rightarrow c_{i, d},
$$

and the associated identification of the Creutz-ladder parameters with the microscopic cold-atom ones:

$t_{h}=+t \mathfrak{\Im}_{2}\left(\frac{V_{d, 0}}{\Delta}\right), \quad \theta=2 \phi_{\uparrow}, \quad t_{d}=-\frac{\Omega}{2} \mathfrak{J}_{0}\left(\frac{V_{d, 0}}{\Delta}\right)$.

In addition, if the Raman beams in Eq. (72) are slightly detuned from the resonance, we would have an additional term,

$$
H_{\text {local }}=\frac{\delta}{2} \sum_{i}\left(f_{i, \uparrow}^{\dagger} f_{i, \uparrow}-f_{i, \downarrow}^{\dagger} f_{i, \downarrow}\right),
$$

where $\delta$ is the Raman detuning, and we work in a rotating frame. By identifying

$$
\Delta \varepsilon=\frac{\delta}{2},
$$

we obtain the last ingredient of the kinetic energy, namely, the leg imbalance in Eq. (5).

So far, we have been concerned with only the kinetic term of the Creutz ladder. As studied for other types of periodic drivings, Refs. [93,94] or Refs. [32,95], the Hubbard interactions present in the microscopic model Eq. (69) may have an important impact in the dressed tunnelings if they modify the resonance conditions. In this work, we assume that such resonances are avoided, which permits mapping the $s$-wave scattering of the cold atoms Eq. (69) onto the required Hubbard interaction of the Creutz ladder Eq. (2) directly:

$$
V_{v}=U_{\uparrow \downarrow}, \quad V_{h}=0 .
$$

We have thus finished with the derivation of the synthetic Creutz-Hubbard model in the cold-atom setup. To summarize, one can explore the properties of the imbalanced Creutz-Hubbard Hamiltonian in Eq. (3) by applying a laser-assisted-tunneling scheme to a two-component gas of fermionic atoms loaded 1D optical lattice, such that the components play the role of the two legs of the ladder [Eq. (78)]. The Hamiltonian parameters can be controlled experimentally using the mappings in Eqs. (79), (80), and (82), together with the expressions (70) and (73), as a guiding principle. The particular Hamiltonian Eq. (3) with $\tilde{t}=t \mathfrak{\Im}_{0}\left(V_{d, 0} / \Delta\right), \Delta \varepsilon=\delta / 2$, and $V_{v}=U_{\uparrow \downarrow}$ is obtained by tuning the ratio of the bare and Raman tunnelings $t / \Omega=-2 \mathfrak{J}_{0}\left(V_{d, 0} / \Delta\right) / \mathfrak{\Im}_{2}\left(V_{d, 0} / \Delta\right)$, such that the strength of the inter- and intraleg tunnelings is equal, and by fixing the phases such that $\phi_{\uparrow}=\pi / 4=-\phi_{\downarrow}$ leads to a net $\pi$ flux.

\section{CONCLUSIONS AND OUTLOOK}

In this work, we advance the understanding of the competition between topological features and interaction effects in quantum many-body systems. By focusing on the paradigmatic Creutz-Hubbard ladder, we develop a variety of analytical tools and perform a thorough numerical analysis, unveiling characteristic features and general methods that could be applied to other strongly correlated topological insulators or superconductors. Moreover, our predictions can be readily tested in tabletop experiments with ultracold fermionic atoms in optical lattices, which may serve as quantum simulators of interacting topological phases.

By using a pair of electronic states of the atoms as synthetic legs of the ladder, and by exploiting laser-assisted tunneling to control their dynamics, we show that the physics of a variant of the Creutz ladder can be implemented in a one-dimensional optical lattice. Such a variant is obtained by applying a Zeeman splitting between the two legs of the ladder, which puts the system into the AIII class of topological insulators. To the best of our knowledge, topological insulators in such a symmetry class have not yet been realized in cold-atom experiments. Moreover, by adding on-site repulsive interactions only, we can induce phase transitions of different universality classes into an orbital ferro- or paramagnetic phase. Surprisingly, we find that the expected Dirac CFT transition line at weak interactions splits into two Majorana CFT critical lines, once the Hubbard term dominates over the Zeeman term: these findings are also numerically supported by the scaling of entanglement entropy. In addition, we provide an understanding of these topological phase transitions in the language of quantum impurity physics, shedding new light on the hybridization mechanism of the edge states. Finally, we identify experimentally accessible signatures to test our theoretical predictions.

From the above results, we are convinced that the synthetic Creutz-Hubbard ladder can become a workhorse in the theoretical and experimental study of correlated 
topological insulators, as it allows for a very neat understanding of the underlying phase diagram and a clear path to implement the model with ultracold atoms. This could be helpful in order to gain even deeper insight into the tricritical point, serving as a guide to construct an effective quantum field theory that describes the mechanism underlying the splitting of the central charge into the two critical lines. It would be very interesting to study the imbalanced Creutz-Hubbard model at different fillings, and to explore the possibility of finding topological phases of matter that disappear for vanishing interactions. In this respect, the analytic and numerical methods hereby presented may be generalized to other fillings, allowing one to go beyond mean-field arguments that support the existence of such interesting ground states.

\section{ACKNOWLEDGMENTS}

We thank L. Mazza and M. Burrello for critical reading of the paper before submission, L. Tarruell for interesting discussions on the experimental details, and L. Tagliacozzo and J. Stasinska for fruitful discussions. J. J. thanks Studienstiftung des deutschen Volkes for financial support. M. R. thanks the KITP Santa Barbara for hospitality within the Visiting Program "Synthetic Quantum Matter," during which part of the manuscript writing was performed. A. B. acknowledges support from Spanish MINECO Project No. FIS2015-70856-P, and CAM PRICYT Project QUITEMAD+S2013/ICE-2801. A. P., S.-J. R., and M. L. acknowledge financial support from Fundació Cellex, from the European Union (ERC-2013-AdG Grant No. 339106 OSYRIS, FP7-ICT-2011-9 No. 600645 SIQS, H2020FETPROACT-2014 No. 641122 QUIC, FP7/2007-2013 Grant No. 323714 Equam), from Spanish MINECO Project (FIS2013-46768-P FOQUS, SEV-2015-0522 Severo Ochoa), from the Generalitat de Catalunya (2014 SGR 874), and from CERCA Programme/Generalitat de Catalunya. Some of the MPS simulations were run by J. J. and M. R. on the Mogon cluster of the JGU (made available by the CSM and AHRP), with a code based on a flexible Abelian Symmetric Tensor Networks Library, developed in collaboration with the group of $\mathrm{S}$. Montangero at the University of Ulm.

[1] X. Wen, From the Origin of Sound to an Origin of Light and Electrons, Quantum Field Theory of Many-Body Systems (Oxford University Press, New York, 2004).

[2] A. Y. Kitaev, Fault-Tolerant Quantum Computation by Anyons, Ann. Phys. (Amsterdam) 303, 2 (2003); C. Nayak, A. Stern, M. Freedman, and S. Das Sarma, Non-Abelian Anyons and Topological Quantum Computation, Rev. Mod. Phys. 80, 1083 (2008).

[3] K. Klitzing, G. Dorda, and M. Pepper, New Method for High-Accuracy Determination of the Fine-Structure
Constant Based on Quantized Hall Resistance, Phys. Rev. Lett. 45, 494 (1980).

[4] D. J. Thouless, M. Kohmoto, M. P. Nightingale, and M. den Nijs, Quantized Hall Conductance in a Two-Dimensional Periodic Potential, Phys. Rev. Lett. 49, 405 (1982).

[5] B. I. Halperin, Quantized Hall Conductance, CurrentCarrying Edge States, and the Existence of Extended States in a Two-Dimensional Disordered Potential, Phys. Rev. B 25, 2185 (1982).

[6] C. L. Kane and M. P. A. Fisher, in Perspectives in Quantum Hall Effects: Novel Quantum Liquids in Low-Dimensional Semiconductor Structures, edited by S. Das Sarma and A. Pinczuk (Wiley-VCH Verlag, Weinheim, 2004).

[7] F. D. M. Haldane, Model for a Quantum Hall Effect without Landau Levels: Condensed-Matter Realization of the "Parity Anomaly", Phys. Rev. Lett. 61, 2015 (1988).

[8] A. Y. Kitaev, Unpaired Majorana Fermions in Quantum Wires, Phys. Usp. 44, 131 (2001).

[9] C. L. Kane and E. J. Mele, $Z_{2}$ Topological Order and the Quantum Spin Hall Effect, Phys. Rev. Lett. 95, 146802 (2005).

[10] A. Altland and M. R. Zirnbauer, Nonstandard Symmetry Classes in Mesoscopic Normal-Superconducting Hybrid Structures, Phys. Rev. B 55, 1142 (1997); A. P. Schnyder, S. Ryu S, A. Furusaki, and A. W. W. Ludwig, Classification of Topological Insulators and Superconductors in Three Spatial Dimensions, Phys. Rev. B 78, 195125 (2008); A. Y. Kitaev, Periodic Table for Topological Insulators and Superconductors, AIP Conf. Proc. 1134, 22 (2009).

[11] See M. Z. Hasan and C. L. Kane, Colloquium: Topological Insulators, Rev. Mod. Phys. 82, 3045 (2010); X.-L. Qi and S.-C. Zhang, Topological Insulators and Superconductors, Rev. Mod. Phys. 83, 1057 (2011), and references therein.

[12] M. König, S.Wiedmann, C. Brüne, A. Roth, H. Buhmann, L. W. Molenkamp, X.-L. Qi, and S.-C. Zhang, Quantum Spin Hall Insulator State in HgTe Quantum Wells, Science 318, 766 (2007).

[13] D. Hsieh, Q. Dong, A. L. Wray, Y. Xia, Y. Hor, R. Cava, and M. Z. Hasan, A Topological Dirac Insulator in a Quantum Spin Hall Phase, Nature (London) 452, 970 (2008).

[14] V. Mourik, K. Zuo, S. M. Frolov, S. R. Plissard, E. P. A. M. Bakkers, and L. P. Kouwenhoven, Signatures of Majorana Fermions in Hybrid Superconductor-Semiconductor Nanowire Devices, Science 336, 1003 (2012).

[15] D. R. Hofstadter, Energy Levels and Wave Functions of Bloch Electrons in Rational and Irrational Magnetic Fields, Phys. Rev. B 14, 2239 (1976).

[16] See M. Hohenadler and F. F. Assaad, Correlation Effects in Two-Dimensional Topological Insulators, J. Phys. Condens. Matter 25, 143201 (2013), and references therein.

[17] R. B. Laughlin, Anomalous Quantum Hall Effect: An Incompressible Quantum Fluid with Fractionally Charged Excitations, Phys. Rev. Lett. 50, 1395 (1983).

[18] See I. Bloch, J. Dalibard, and W. Zwerger, Many-Body Physics with Ultracold Gases, Rev. Mod. Phys. 80, 885 (2008), and references therein.

[19] See M. Lewenstein, A. Sanpera, V. Ahufinger, B. Damski, A. Sen, and U. Sen, Ultracold Atomic Gases in Optical Lattices: Mimicking Condensed Matter Physics and Beyond, Adv. Phys. 56, 243 (2007), and references therein. 
[20] See I. Bloch, J. Dalibard, and S. Nascimbène, Quantum Simulations with Ultracold Quantum Gases, Nat. Phys. 8, 267 (2012), and references therein.

[21] M. P. A. Fisher, P. B. Weichman, G. Grinstein, and D. S. Fisher, Boson Localization and the Superfluid-Insulator Transition, Phys. Rev. B 40, 546 (1989).

[22] D. Jaksch, C. Bruder, J. I. Cirac, C. W. Gardiner, and P. Zoller, Cold Bosonic Atoms in Optical Lattices, Phys. Rev. Lett. 81, 3108 (1998).

[23] M. Greiner, O. Mandel, T. Esslinger, T. W. Hänsch, and I. Bloch, Quantum Phase Transition from a Superfluid to a Mott Insulator in a Gas of Ultracold Atoms, Nature (London) 415, 39 (2002).

[24] J. Hubbard, Electron Correlations in Narrow Energy Bands, Proc. R. Soc. A 276, 238 (1963).

[25] W. Hofstetter, J. I. Cirac, P. Zoller, E. Demler, and M. D. Lukin, High-Temperature Superfluidity of Fermionic Atoms in Optical Lattices, Phys. Rev. Lett. 89, 220407 (2002).

[26] R. Jördens, N. Strohmaier, K. Günter, H. Moritz, and T. Esslinger, A Mott Insulator of Fermionic Atoms in an Optical Lattice, Nature (London) 455, 204 (2008); U. Schneider, L. Hackermüller, S. Will, Th. Best, I. Bloch, T. A. Costi, R. W. Helmes, D. Rasch, and A. Rosch, Metallic and Insulating Phases of Repulsively Interacting Fermions in a 3D Optical Lattice, Science 322, 1520 (2008).

[27] D. Jaksch and P. Zoller, Creation of Effective Magnetic Fields in Optical Lattices: The Hofstadter Butterfly for Cold Neutral Atoms, New J. Phys. 5, 56 (2003).

[28] N. Goldman, I. Satija, P. Nikolic, A. Bermudez, M. A. Martin-Delgado, M. Lewenstein, and I. B. Spielman, Realistic Time-Reversal Invariant Topological Insulators with Neutral Atoms, Phys. Rev. Lett. 105, 255302 (2010).

[29] K. Wilson, in New Phenomena in Subnuclear Physics, edited by A. Zichichi (Plenum, New York, 1977).

[30] See N. Goldman, G. Juzeliunas, P. Öhberg, and I. B. Spielman, Light-Induced Gauge Fields for Ultracold Atoms, Rep. Prog. Phys. 77, 126401 (2014), and references therein.

[31] See N. Goldman, J. C. Budich, and P. Zoller, Topological Quantum Matter with Ultracold Gases in Optical Lattices, Nat. Phys. 12, 639 (2016), and references therein.

[32] M. Aidelsburger, M. Atala, M. Lohse, J. T. Barreiro, B. Paredes, and I. Bloch, Realization of the Hofstadter Hamiltonian with Ultracold Atoms in Optical Lattices, Phys. Rev. Lett. 111, 185301 (2013); H. Miyake, G. A. Siviloglou, C. J. Kennedy, W. C. Burton, and W. Ketterle, Realizing the Harper Hamiltonian with Laser-Assisted Tunneling in Optical Lattices, Phys. Rev. Lett. 111, 185302 (2013); M. Atala, M. Aidelsburger, M. Lohse, J. T. Barreiro, B. Paredes, and I. Bloch, Observation of Chiral Currents with Ultracold Atoms in Bosonic Ladders, Nat. Phys. 10, 588 (2014); M. Aidelsburger, M. Lohse, C. Schweizer, M. Atala, J. T. Barreiro, S. Nascimbene, N. R. Cooper, I. Bloch, and N. Goldman, Measuring the Chern Number of Hofstadter Bands with Ultracold Bosonic Atoms, Nat. Phys. 11, 162 (2015).

[33] G. Jotzu, M. Messer, R. Desbuquois, M. Lebrat, T. Uehlinger, D. Greif, and T. Esslinger, Experimental Realization of the Topological Haldane Model with Ultracold Fermions, Nature (London) 515, 237 (2014); N. Fläschner, B. S. Rem, M. Tarnowski, D. Vogel, D.-S. Lühmann,
K. Sengstock, and C. Weitenberg, Experimental Reconstruction of the Berry Curvature in a Floquet Bloch Band, Science 352, 1091 (2016).

[34] C. Chin, R. Grimm, P. Julienne, and E. Tiesinga, Feshbach Resonances in Ultracold Gases, Rev. Mod. Phys. 82, 1225 (2010).

[35] M. Creutz, End States, Ladder Compounds, and DomainWall Fermions, Phys. Rev. Lett. 83, 2636 (1999).

[36] See U. Schollwoeck, The Density-Matrix Renormalization Group in the Age of Matrix Product States, Ann. Phys. (Amsterdam) 326, 96 (2011), and references therein.

[37] W. P. Su, J. R. Schrieffer, and A. J. Heeger, Solitons in Polyacetylene, Phys. Rev. Lett. 42, 1698 (1979).

[38] M. Atala, M. Aidelsburger, J. T. Barreiro, D. Abanin, T. Kitagawa, E. Demler, and I. Bloch, Direct Measurement of the Zak Phase in Topological Bloch Bands, Nat. Phys. 9, 795 (2013).

[39] M. Creutz and I. Horváth, Surface States and Chiral Symmetry on the Lattice, Phys. Rev. D 50, 2297 (1994).

[40] D. B. Kaplan, A Method for Simulating Chiral Fermions on the Lattice, Phys. Lett. B 288, 342 (1992).

[41] H. B. Nielsen and M. Ninomiya, Absence of Neutrinos on a Lattice: (I). Proof by Homotopy Theory, Nucl. Phys. B185, 20 (1981); Absence of Neutrinos on a Lattice: (II). Intuitive Topological Proof, Nucl. Phys. B193, 173 (1981).

[42] A. Bermudez, L. Mazza, M. Rizzi, N. Goldman, M. Lewenstein, and M. A. Martin-Delgado, Wilson Fermions and Axion Electrodynamics in Optical Lattices, Phys. Rev. Lett. 105, 190404 (2010).

[43] See D. Xiao, M.-C. Chang, and Q.Niu, Berry Phase Effects on Electronic Properties, Rev. Mod. Phys. 82, 1959 (2010), and references therein.

[44] J. Zak, Berry's Phase for Energy Bands in Solids, Phys. Rev. Lett. 62, 2747 (1989).

[45] M. Tovmasyan, E. van Nieuwenburg, and S. D. Huber, Geometry-Induced Pair Condensation, Phys. Rev. B 88, 220510(R) (2013).

[46] S. Takayoshi, H. Katsura, N. Watanabe, and H. Aoki, Phase Diagram and Pair Tomonaga-Luttinger Liquid in a BoseHubbard Model with Flat Bands, Phys. Rev. A 88, 063613 (2013).

[47] S. D. Huber and E. Altman, Bose Condensation in Flat Bands, Phys. Rev. B 82, 184502 (2010).

[48] M. Tovmasyan, S. Peotta, P. Törmä, and S. D. Huber, Effective Theory and Emergent SU(2) Symmetry in the Flat Bands of Attractive Hubbard Models, Phys. Rev. B 94, 245149 (2016).

[49] D. Sticlet, L. Seabra, F. Pollmann, and J. Cayssol, From Fractionally Charged Solitons to Majorana Bound States in a One-Dimensional Interacting Model, Phys. Rev. B 89, 115430 (2014).

[50] L. Mazza, M. Aidelsburger, H.-H. Tu, N. Goldman, and M. Burrello, Methods for Detecting Charge Fractionalization and Winding Numbers in an Interacting Fermionic Ladder, New J. Phys. 17, 105001 (2015).

[51] See S. A. Parameswaran, R. Roy, and S. L. Sondhi, Fractional Quantum Hall Physics in Topological Flat, C.R. Phys. 14, 816 (2013), and references therein.

[52] D. A. Abanin, T. Kitagawa, I. Bloch, and E. Demler, Interferometric Approach to Measuring Band Topology 
in 2D Optical Lattices, Phys. Rev. Lett. 110, 165304 (2013); F. Grusdt, D. Abanin, and E. Demler, Measuring $\mathbb{Z}_{2}$ Topological Invariants in Optical Lattices Using Interferometry, Phys. Rev. A 89, 043621 (2014).

[53] J. Vidal, R. Mosseri, and B. Doucot, Aharonov-Bohm Cages in Two-Dimensional Structures, Phys. Rev. Lett. 81, 5888 (1998).

[54] M. Mancini, G. Pagano, G. Cappellini, L. Livi, M. Rider, J. Catani, C. Sias, P. Zoller, M. Inguscio, M. Dalmonte, and L. Fallani, Observation of Chiral Edge States with Neutral Fermions in Synthetic Hall Ribbons, Science 349, 1510 (2015).

[55] B. K. Stuhl, H.-I. Lu, L. M. Aycock, D. Genkina, and I. B. Spielman, Visualizing Edge States with an Atomic Bose Gas in the Quantum Hall Regime, Science 349, 1514 (2015).

[56] A. L. Gaunt, T. F. Schmidutz, I. Gotlibovych, R. P. Smith, and Z. Hadzibabic, Bose-Einstein Condensation of Atoms in a Uniform Potential, Phys. Rev. Lett. 110, 200406 (2013); A. L. Navon, A. L. Gaunt, R. P. Smith, and Z. Hadzibabic, Critical Dynamics of Spontaneous Symmetry Breaking in a Homogeneous Bose Gas, Science 347, 167 (2015); L. Corman, L. Chomaz, T. Bienaimé, R. Desbuquois, C. Weitenberg, S. Nascimbene, J. Dalibard, and J. Beugnon, Quench-Induced Supercurrents in an Annular Bose Gas, Phys. Rev. Lett. 113, 135302 (2014).

[57] N. Goldman, J. Beugnon, and F. Gerbier, Detecting Chiral Edge States in the Hofstadter Optical Lattice, Phys. Rev. Lett. 108, 255303 (2012).

[58] T. D. Stanescu, V. Galitski, and S. Das Sarma, Topological States in Two-Dimensional Optical Lattices, Phys. Rev. A 82, 013608 (2010).

[59] E. Fradkin and L. Susskind, Order and Disorder in Gauge Systems and Magnets, Phys. Rev. D 17, 2637 (1978); J. B. Kogut, An Introduction to Lattice Gauge Theory and Spin Systems, Rev. Mod. Phys. 51, 659 (1979).

[60] P. Jordan and E. Wigner, Über das Paulische Äquivalenzverbot, Z. Phys. 47, 631 (1928).

[61] J. H. Taylor and G. Müller, Magnetic Field Effects in the Dynamics of Alternating or Anisotropic Quantum Spin Chains, Physica (Amsterdam) 130A, 1 (1985).

[62] F. Grusdt, N. Y. Yao, D. Abanin, M. Fleischhauer, and E. Demler, Interferometric Measurements of Many-Body Topological Invariants Using Mobile Impurities, Nat. Commun. 7, 11994 (2016).

[63] I. Carusotto, Bragg Scattering and the Spin Structure Factor of Two-Component Atomic Gases, J. Phys. B 39, S211 (2006).

[64] J. H. Drewes, L. A. Miller, E. Cocchi, C. F. Chan, N. Wurz, M. Gall, D. Pertot, F. Brennecke, and M. Köhl, Antiferromagnetic Correlations in Two-Dimensional Fermionic Mott-Insulating and Metallic Phases, Phys. Rev. Lett. 118, 170401 (2017).

[65] K. Eckert, O. Romero-Isart, M. Rodriguez, M. Lewenstein, E. S. Polzik, and A. Sanpera, Quantum Non-Demolition Detection of Strongly Correlated Systems, Nat. Phys. 4, 50 (2008).

[66] D. Greif, M. F. Parsons, A. Mazurenko, C. S. Chiu, S. Blatt, F. Huber, G. Ji, and M. Greiner, Site-Resolved Imaging of a Fermionic Mott Insulator, Science 351, 953 (2016); M. Boll, T. A. Hilker, G. Salomon, A. Omran, J. Nespolo,
L. Pollet, I. Bloch, and C. Gross, Spin- and DensityResolved Microscopy of Antiferromagnetic Correlations in Fermi-Hubbard Chains, Science 353, 1257 (2016).

[67] P. W. Anderson, New Approach to the Theory of Superexchange Interactions, Phys. Rev. 115, 2 (1959).

[68] L.-M. Duan, E. Demler, and M. D. Lukin, Controlling Spin Exchange Interactions of Ultracold Atoms in Optical Lattices, Phys. Rev. Lett. 91, 090402 (2003).

[69] S. Trotzky, P. Cheinet, S. Fölling, M. Feld, U. Schnorrberger, A. M. Rey, A. Polkovnikov, E. A. Demler, M. D. Lukin, and I. Bloch, Time-Resolved Observation and Control of Superexchange Interactions with Ultracold Atoms in Optical Lattices, Science 319, 295 (2008).

[70] D. Greif, T. Uehlinger, G. Jotzu, L. Tarruell, and T. Esslinger, Short-Range Quantum Magnetism of Ultracold Fermions in an Optical Lattice, Science 340, 1307 (2013); R. A. Hart, P. M. Duarte, T.-L. Yang, X. Liu, T. Paiva, E. Khatami, R. T. Scalettar, N. Trivedi, D. A. Huse, and R. G. Hulet, Observation of Antiferromagnetic Correlations in the Hubbard Model with Ultracold Atoms, Nature (London) 519, 211 (2015).

[71] A. H. MacDonald, S. M. Girvin, and D. Yoshioka, $t / U$ Expansion for the Hubbard Model, Phys. Rev. B 37, 9753 (1988).

[72] P. Pfeuty, The One-Dimensional Ising Model with a Transverse Field, Ann. Phys. (N.Y.) 57, 79 (1970).

[73] N. N. Bogoliubov, On a New Method in the Theory of Superconductivity, Sov. Phys. JETP, 7, 41 (1958) [Nuovo Cimento Soc. Ital. Fis. 6, 794 (1958)].

[74] See O. Dutta, M. Gajda, P. Hauke, M. Lewenstein, D.-S. Lhmann, B. A. Malomed, T. Sowiski, and J. Zakrzewski, Non-Standard Hubbard Models in Optical Lattices: A Review, Rep. Prog. Phys. 78, 066001 (2015), and references therein.

[75] The energies of the bulk bands for the finite chain can differ from those of the periodic chain by an intensive quantity related to the missing bond $t_{\text {imb }}$ connecting the edges. In the thermodynamic limit $N \rightarrow \infty$, this difference will be irrelevant for any extensive observable, or any quantity that depends on a sum over all possible bulk energies.

[76] P. W. Anderson, Localized Magnetic States in Metals, Phys. Rev. 124, 41 (1961); U. Fano, Effects of Configuration Interaction on Intensities and Phase Shifts, Phys. Rev. 124, 1866 (1961).

[77] N. N. Bogoliubov, On a New Method in the Theory of Superconductivity, Sov. Phys. JETP, 7, 41 (1958) [Nuovo Cimento Soc. Ital. Fis. 6, 794 (1958)].

[78] See L. Amico, R. Fazio, A. Osterloh, and V. Vedral, Entanglement in Many-Body Systems, Rev. Mod. Phys. 80, 517 (2008), and references therein.

[79] T. Osborne and M. Nielsen, Entanglement in a Simple Quantum Phase Transition, Phys. Rev. A 66, 032110 (2002); A. Osterloh, L. Amico, G. Falci, and R. Fazio, Scaling of Entanglement Close to a Quantum Phase Transition, Nature (London) 416, 608 (2002).

[80] G. Vidal, J. Latorre, E. Rico, and A. Kitaev, Entanglement in Quantum Critical Phenomena, Phys. Rev. Lett. 90, 227902 (2003); P. Calabrese and J. Cardy, Entanglement Entropy and Quantum Field Theory, J. Stat. Mech. (2004) P06002. 
[81] See P. Calabrese and J. Cardy, Entanglement Entropy and Conformal Field Theory, J. Phys. A 42, 504005 (2009), and references therein.

[82] H. Li and F. D. M. Haldane, Entanglement Spectrum as a Generalization of Entanglement Entropy: Identification of Topological Order in Non-Abelian Fractional Quantum Hall Effect States, Phys. Rev. Lett. 101, 010504 (2008).

[83] F. Pollmann, A. M. Turner, E. Berg, and M. Oshikawa, Entanglement Spectrum of a Topological Phase in One Dimension, Phys. Rev. B 81, 064439 (2010).

[84] A. Bermudez, D. Patane, L. Amico, and M. A. MartinDelgado, Topology-Induced Anomalous Defect Production by Crossing a Quantum Critical Point, Phys. Rev. Lett. 102, 135702 (2009).

[85] L. Mazza, A. Bermudez, N. Goldman, M. Rizzi, M. A. Martin-Delgado, and M. Lewenstein, An Optical-LatticeBased Quantum Simulator for Relativistic Field Theories and Topological Insulators, New J. Phys. 14, 015007 (2012).

[86] O. Boada, A. Celi, M. Lewenstein, and J. I. Latorre, Quantum Simulation of an Extra Dimension, Phys. Rev. Lett. 108, 133001 (2012).

[87] A. Celi, P. Massignan, J. Ruseckas, N. Goldman, I. B. Spielman, G. Juzeliunas, and M. Lewenstein, Synthetic Gauge Fields in Synthetic Dimensions, Phys. Rev. Lett. 112, 043001 (2014).

[88] See A. Eckardt, Colloquium: Atomic Quantum Gases in Periodically Driven Optical Lattices, Rev. Mod. Phys. 89, 011004 (2017), and references therein.

[89] A. Bermudez, T. Schätz, and D. Porras, Synthetic Gauge Fields for Vibrational Excitations of Trapped Ions, Phys. Rev. Lett. 107, 150501 (2011); P. Hauke, O. Tieleman,
A. Celi, C. Ölschläger, J. Simonet, J. Struck, M. Weinberg, P. Windpassinger, K. Sengstock, M. Lewenstein, and A. Eckardt, Non-Abelian Gauge Fields and Topological Insulators in Shaken Optical Lattices, Phys. Rev. Lett. 109, 145301 (2012).

[90] F. Gerbier and J. Dalibard, Gauge Fields for Ultracold Atoms in Optical Superlattices, New J. Phys. 12, 033007 (2010).

[91] G. Jotzu, M. Messer, F. Görg, D. Greif, R. Desbuquois, and T. Esslinger, Creating State-Dependent Lattices for Ultracold Fermions by Magnetic Gradient Modulation, Phys. Rev. Lett. 115, 073002 (2015).

[92] K. Drese and M. Holthaus, Ultracold Atoms in Modulated Standing Light Waves, Chem. Phys. 217, 201 (1997); C. Sias, H. Lignier, Y. P. Singh, A. Zenesini, D. Ciampini, O. Morsch, and E. Arimondo, Observation of Photon-Assisted Tunneling in Optical Lattices, Phys. Rev. Lett. 100, 040404 (2008).

[93] R. Ma, M. E. Tai, P. M. Preiss, W. S. Bakr, J. Simon, and M. Greiner, Photon-Assisted Tunneling in a Biased Strongly Correlated Bose Gas, Phys. Rev. Lett. 107, 095301 (2011); Y.-A. Chen, S. Nascimbene, M. Aidelsburger, M. Atala, S. Trotzky, and I. Bloch, Controlling Correlated Tunneling and Superexchange Interactions with ac-Driven Optical Lattices, Phys. Rev. Lett. 107, 210405 (2011).

[94] A. J. Daley and J. Simon, Effective Three-Body Interactions via Photon-Assisted Tunneling in an Optical Lattice, Phys. Rev. A 89, 053619 (2014).

[95] A. Bermudez and D. Porras, Interaction-Dependent Photon-Assisted Tunneling in Optical Lattices: A Quantum Simulator of Strongly-Correlated Electrons and Dynamical Gauge Fields, New J. Phys. 17, 103021 (2015). 